\title{
PATTERN OF ACUTE POISONING AND GENDER COMPARISON AMONG CASES ADMITTED TO BENHA POISON CONTROL UNIT: A FIVE YEAR RETROSPECTIVE- DESCRIPTIVE STUDY (2011-2015)
}

\author{
Mohamed F. Khodeary ${ }^{1,2}$ and Shereen M. S. Elkholy ${ }^{1}$ \\ ${ }^{1}$ Department of Forensic Medicine and Clinical Toxicology, Faculty of Medicine, \\ Benha University, \\ ${ }^{2}$ Benha Poison Control Unit, Benha University Hospitals
}

\begin{abstract}
Background: Acute poisoning is a growing healthcare problem and an important cause of considerable morbidity and mortality worldwide, including Egypt. Its incidence and characteristics varies significantly between countries and different regions within the same country. Aim: This retrospective-descriptive study was conducted at Benha Poison Control Unit over a period of 5-year (2011-2015) to identify and analyze the characteristics of poisoned cases with special reference to comparison between gender. Research Methodology: Toxicological records were reviewed for socio-epidemiological, toxicological, implicated poisonous substances, clinical findings, and applied therapeutic modalities. Results: The study included 4372 case. The majority of overall cases were females (58.37\%), under 18-year (67.86\%), children <6-year $(38.88 \%)$ and adults between 18-29-year (17.77\%), unmarried (85.36\%), rural residents (70.08\%), of low economic (67.77\%) and educational status $(48.86 \%)$, and unemployed (54.19\%). Most patients showed poisoning at homes (92.02\%), a pre-hospitalization period for 3-hour (42.54\%), hospitalized during afternoon $(48.47 \%)$ and summer $(35.18 \%)$ times as well as exposures via oral route $(84.95 \%)$, unintentional manner (55.79\%), and single agent (92.78\%). Poisoning with non-medicinal $(54.6 \%)$ predominated over medicinal (45.4\%) products and the most common involved groups were, respectively, pesticides $(25.14 \%)$ and corrosives $(9.49 \%)$ and sedative-hypnotics $(8.55 \%)$ and non-opioid analgesics $(5.63 \%)$. The most frequently recorded clinical findings were mild toxidromes $(77.13 \%)$, emergency room admission $(70.31 \%)$, and hospitalization for $\leq 1$-day (73.19\%), complete recovery $(97.71 \%)$ with low mortality rate $(0.85 \%)$, and organophosphates were the chief cause of death $(0.32 \%)$. Therapeutic interventions mostly involved internal decontamination $(64.75 \%)$ by administration of activated charcoal $(36.37 \%)$ and performance of gastric lavage $(22.62 \%)$, followed by administration of specific antidotes $(25.25 \%)$. The proportions of males were higher than females regarding age groups $<18$-year and children <6-year, low economic level, unintentional manner, medicinal preparations, mortality rate, death by organophosphates, management with internal decontamination, activated charcoal, gastric lavage, and specific antidotes. Whereas, the proportions of females were higher than males regarding age group between 18-29year, unmarried, rural residents, low educational level, poisoning at homes, afternoon and summer times, non-medicinal substances, pesticides and corrosives, non-opioid analgesics, mild toxidromes, emergency room admission, and complete recovery. There were statistically significant differences between genders regarding the
\end{abstract}


aforementioned variables. Conclusion: The current findings provide some data about the magnitude of acute poisoning in Benha region and its surrounding areas. Community education about the danger of poisonous substances and understanding the motivations behind this problem are recommended to reduce the incidence of poisoning.

Keywords: acute poisoning, gender differences, epidemiology, medicinal-related poisoning, non-medicinal-related poisoning.

\section{INTRODUCTION}

Acute poisoning by vast numbers of various poisonous substances is an extremely significant, widespread, and growing dilemma all over the world (Srivastava et al., 2005; Moazzam et al., 2009) and has become a major and serious health problem facing both developed (Räsänen et al., 2002; Mowry et al., 2015) and developing countries (Sawalha et al., 2010; Jaiprakash et al., 2011), including Egypt (El Masry and Tawfik, 2013; Hassan and Siam, 2014). The costs of medical management can be substantial, leading to considerable negative implications on the medico-social services that expend or reduce their valuable resources besides adding further massive economic burden on the community (Afshari et al., 2004; Rajapakse et al., 2014).

This global issue can be attributed to the exponential growth in the number and types of new substances production as a result of rapid industrialization and technological advancement in the fields of agriculture, industrial technology, and medical pharmacy. Hence, extensive availability and progressive flooding of the global markets with such enormous substances can increase the risk of human exposure to poisons (Shadnia et al., 2007; Ahmadi et al., 2010). Toxic substances are plenty and involve synthetic toxicants such as pharmaceutical or medicinal products and non-pharmaceutical or chemical compounds (pesticides, household cleaning substances, fertilizers, petroleum distillates) and natural toxins (venomous animals, toxic plants, pathologic food contaminants, poisonous fish) as well as ayurvedic and homeopathic products (Lall et al., 2003; Mowry et al., 2015). Therefore, identification of hazardous substances may not be straightforward and can add more burdens on the healthcare providers.

Acute poisoning is not only one of the most important reasons of emergency hospital visits and/or admissions (Tüfekçi et al., 2004; Ahmadi et al., 2010), but also a leading cause of considerable unnatural morbidity and mortality worldwide (Islambulchilar et al., 2009; Khudair et al., 2013), including Egypt (Taha et al., 2011; Abdel Moneim et al., 2012; Aboul-Hagag et al., 2013; El Masry and Tawfik, 2013; Hassan and Siam, 2014).

The prevalence rates of acute poisoning significantly vary between countries. According to the American Association of Poison Control Centers' National Poison Data System, approximately 62.3 million human cases suffered poison exposures over a 32 years from 1983 to 2014 with an annual incidence of both unintentional and intentional human poisoning varies between 5.8 to 6.7 poisoned cases per 1000 inhabitants (Mowry et al., 2015). Furthermore, in Egypt, Poison Control 
Center of Ain Shams University was engaged in management of 21550 intoxicated cases during the year 2011 (EI Masry and Tawfik, 2013). Also, in Iran (Hassanian-Moghaddam et al., 2014; Azizpour et al., 2016) and Malaysia (Rajasuriar et al., 2007) several thousands of poisoned cases were admitted to healthcare facilities.

According to the Global Burden of Disease study of the World Health Organization, in 2004 an estimated 346000 individuals died globally from unintentional poisoning and about $91 \%$ of these deaths occurred in low- and medium-income countries. Based on the disability-adjusted life years, poisoning is responsible for loss of over 7.4 million years of healthy life (Mathers et al., 2008). In the United States, unintentional and intentional exposures to poisons resulted in 1173 human fatalities in the year 2014 (Mowry et al., 2015), while in Egypt 2011, the recorded mortality rate due to poisoning was $0.3 \%$ (61 cases) in the year 2011 (El Masry and Tawfik, 2013). Also, the Iranian study of HassanianMoghaddamet al. (2014) showed 2109 deceased cases due to poisoning between the years 2006 to 2011. Despite this, the overall incidences of the acute poisoning can be higher because it is remarkably difficult to determine the exact number of poisoning associated morbidity and mortality due to either underestimated and unreported cases or lack of Poison Control Center consultation and surveillance (Shadnia et al., 2007; Obeidat et al., 2010), even in countries with advanced health care systems for data collection (Mowry et al., 2015).

The incidence and characteristics of acute poisoning varies widely between countries and depends on several diverse and complicated factors such as industrialized and urbanized levels, agriculture style, the availability of diagnostic and therapeutic healthcare facilities and expertise consultations (Lall et al., 2003), accessibility and availability of potentially poisonous substances, socio-economic conditions, cultural background and faith/religious beliefs (Afshari et al., 2004; El Masry and Tawfik, 2013), and legal reprisal or liability (Hatzitolios et al., 2001).

Authoritative data regarding the pattern of poisoning in Benha (the capital of Al-Qalyubia province, north of Cairo, Egypt) and surrounding areas are sparse and incomplete. Based on the acute poisoning admissions to Benha Poison Control Unit, this five-year (2011-2015) retrospective-descriptive study was conducted to identify and analyze the pattern of acute poisoning and gender differences of the overall cases in order to understand the magnitude of this problem that may help in minimizing exposure to poisonous agents and to establish an appropriate strategy for early diagnosis and treatment of poisoned patients, which in turn may lead to reduction of poisoning associated morbidity and mortality.

\section{RESEARCH METHODOLOGY}

Benha Poison Control Unit
$\{B P C U\}$, a part of Benha University Hospitals, Benha, Al-Qalyubia, Egypt, is under supervision of medical staff of Forensic Medicine and Clinical Toxicology Department as well as paramedical high nurses who runs the unit 24 hours per day, 7 days per week. This five-year retrospective study $\left(1^{\text {st }}\right.$ of January 2011 to the $31^{\text {th }}$ of December 2015) was designated to compile and analyze socio-epidemiological, toxicological, implicated poisonous 
substances, clinical findings, and applied therapeutic data of poisoned cases who admitted to BPCU. The data were obtained by reviewing toxicological medical records of the unit and each item was described separately (i.e. no correlations were carried out between the overall recorded parameters). The study was approved by the Ethics Committee of Scientific Research, Faculty of Medicine, Benha University, Egypt.

Inclusion criteria: All poisoned cases who had admitted to BPCU due to poisoning by medicinal and nonmedicinal compounds were included. Exclusion criteria: Poisoned patients as a result of pathogenic food poisoning (food contaminated with various infectious organisms such as bacteria, parasites, or viruses) were excluded from the study.

Regarding the socioepidemiological pattern, the compiled information included the following: gender, age (<18-year [<6, 7-12, 13-15, and 16-<18] and $\geq 18$-year [18-29, 3039, 40-49, and >50]), marital status (married or unmarried as single, engagement breakdown, divorced, widow), residency (rural or urban), income level of families or adult persons (low, middle, or high), educational level (low as illiterates and those registered in or graduated from primary, middle, and secondary schools, or high as those registered in or graduated from university), occupational status (employed as governmental, nongovernmental, household, manual, professional, and agricultural workers, and other jobs, or unemployed as jobless, housewives, and students), and previous health status (disability e.g. mental and physical, other concomitant diseases e.g. diabetes mellitus, hypertension,

cardiovascular, pulmonary, hepato-renal, or other diseases, or healthy).

Concerning the toxicological profile, the collected data involved the following: poisoning scene location (home or other places as street, work place), pre-hospital period (time delay or lag from exposure to hospital arrival), hospital admission shifts (morning, afternoon, evening, or night hours), seasonal variations (summer, spring, autumn, or winter), route of exposure (oral, pulmonary, skin puncture by bites, stings, and injections, dermal contact, or snorting), manner of poisoning (intentional, unintentional, or homicidal), the number of cases per group of substance (medicinal or nonmedicinal), the number of cases per number of substances (single or multiple for both medicinal and non-medicinal groups), and the actual number of implicated poisonous agents (sum of all single and all multiple agents for both medicinal and non-medicinal groups as well as for each group).

Based on the International Classification of Diseases, version 10Clinical Modification (an online ICD10-CM), the types of implicated poisonous agents were classified into two main categories; medicinal (ICD-10 diagnosis codes T36-T50; www.icd10data.com/ICD10CM/Codes/ S00-T88/T36-T50) and non-medicinal (ICD-10 diagnosis codes T51-T65; www.icd10data.com/ICD10CM/Codes/ S00-T88/T51-T65) substances. Also, the most common involved medicinal and non-medicinal causative agents were categorized

(www.icd10data.com/ICD10CM/Table_ Of_Drugs).

Concerning the clinical findings, the clinical severity of each case was 
categorized into one of five grades according to Poisoning Severity Score (PSS) criteria of Persson et al.(1998) as follows: (0)-none (no symptoms or signs related to poisoning), (1)-mild (minor transient and spontaneously resolving symptoms), (2)-moderate (pronounced or prolonged symptoms), (3)-severe (intense or life-threatening symptoms), or (4)-fatal (death). Additionally, ward admission (emergency room, inpatient, or intensive care unit), the hospital stay $(\leq 1,2,3,4$, or $\geq 5$ days), final outcome (complete recovery or with nonfatal sequelae), and substances-induced death.

The main therapeutic interventions included utilizations of external and/or internal decontamination (single or two methods interventions with activated charcoal, gastric lavage, and laxative as well as the use of demulcents), specific antidotes, symptomatic treatment, and emergent and supportive measures.

The collected data were organized, tabulated, and analyzed. Microsoft Excel (2000 Microsoft Corporation, USA) was used to perform descriptive statistics, while 2-sample z-test (an online EpiTools $^{\circledR}$ epidemiological calculator) was used to compare proportions between genders. A calculated two-tailed P-values at or less than $0.05(<0.05)$ were considered as statistically significant.

\section{RESULTS}

A total of 4708 intoxicated cases were admitted to BPCU during the fiveyear study period (2011-2015). However, a total of $336(7.14 \%)$ cases were excluded from the study (199 cases accounted for $4.23 \%$; 115 males and 84 females; had incomplete data or referred to other Poison Control Centers in Egypt and 137 cases accounted for $3.14 \%$; 51 males and 86 females; had pathogenic food poisoning), leaving a study sample of 4372 patients (an annual average of about 875 cases per year) with sufficient information.

Table (1) illustrates the socioepidemiological distribution pattern. The vast majority of the overall cases were females $(58.37 \%)$ with a sex ratio of 1.4:1, under 18-year old $(67.86 \%)$, unmarried $(85.36 \%)$, rural residents (70.08\%), of low economic (67.77\%) and educational (48.86\%) status, unemployed $(54.19 \%)$, and previously healthy $(77.45 \%)$. Also, poisoning events were predominantly seen among both children <6-year (38.88\%) and adults between 18-29-year (17.77\%) and household workers (22.92\%).

The distribution patterns between genders showed higher proportion of males than females in terms of variables listed in the age groups <18-year $(75.77 \%)$ and <6-year $(56.1 \%)$, married status (20.27\%), urban residence (40.27\%), low income level (69.95\%), professional $(6.26 \%)$ and agricultural occupations (5\%), and mental and physical disabilities (7.64\%). Whereas, the proportions of females were more prevalently recorded than males regarding variables categorized in almost all age groups ( 7 to $>50$ years, especially $>18$-year; $37.77 \%$ and $18-29$ year; $19.79 \%$ ), unmarried status (89.38\%), rural residence $(77.47 \%)$, middle income level (22.84\%) and all educational levels (low: 57.01\% and high: $16.38 \%$ ), non-governmental $(5.96 \%)$ and household (25.9\%) jobs, and the presence of other concomitant diseases (19.28\%). Statistical analysis revealed significant differences between genders in relation to the aforementioned variables $(\mathrm{P}<0.05)$.

Table (2) shows the distribution 
pattern of toxicological data. Among the overall cases, the most common reported findings were poisoning at homes (92.02\%), pre-hospitalization period for 3-hour (42.54\%), hospital admission during afternoon (48.47\%) and evening $(26.05 \%)$ shifts as well as during summer $(35.18 \%)$ and spring $(31.45 \%)$ seasons, exposure via oral route $(84.95 \%)$, unintentional manner of exposure $(55.79 \%)$, and exposure to single agent $(92.68 \%)$. Additionally, the overall percentage of implicated poisonous agents $(111.07 \%$; sum of all single and all multiple agents for both medicinal and non-medicinal groups, especially the implicated non-medicinal agents; 57.69\%), slightly exceeded that of the overall intoxicated cases (as some patients had exposed to more than one substance) and poisoning with multiple medicinal agents (12.58\%) was more common than non-medicinal agents $(5.81 \%)$.

The affected males were higher than females as regards parameters assorted in poisoning at other places (14.12\%), pre-hospitalization period for 2-hour (19.23\%), hospital admission during evening (43.24\%) and night (15.44\%) shifts as well as during spring months $(49.45 \%)$, skin $(4.67 \%)$ and snorting $(1.15 \%)$ routes of exposure, unintentional manner of exposure (70.77\%), all implicated medicinal poisonous agents (59.4\%), and all involved multiple substances (20\%), especially multiple non-medicinal substances $(8.35 \%)$. While, the affected females were more predominant than males as regards parameters assorted in poisoning at homes $(96.39 \%)$, prehospitalization period for 4-hour (25.59\%), hospital admission during afternoon $(60.93 \%)$ and morning $(18.89 \%)$ shifts as well as during summer (41.22\%), autumn (25.43\%), and winter $(14.73 \%)$ seasons, dermal route of exposure $(3.06 \%)$, intentional manner of exposure $(54.9 \%)$, and all implicated non-medicinal poisonous agents (60.89\%). There were statistically significant variances among genders in respect to the aforesaid variables $(\mathrm{P}<0.05)$.

Table (3) displays the distribution pattern of poisonous medicinal and nonmedicinal groups based on the ICD-10 classification. Regarding the overall pattern of poisonous groups, the prevalence of poisoning with nonmedicinal group $(54.6 \%)$ was higher than medicinal group $(45.4 \%)$ and exposure to single non-medicinal agent $(51.88 \%)$ predominated over single medicinal agent $(40.81 \%)$, whereas poisoning with multiple medicinal $(4.6 \%)$ was more common than multiple non-medicinal agents $(2.72 \%)$.

Exposure to single medicinal (47.75\%), all medicinal (single and multiple) $(51.59 \%)$, and multiple nonmedicinal substances $(3.57 \%)$ were frequently noticed among males. Whereas, exposure to multiple medicinal $(5.13 \%)$, single nonmedicinal $(56.9 \%)$, and all nonmedicinal $(59.01 \%)$ agents were commonly seen among females. There were statistically significant distinctions among genders in relation to the aforementioned variables $(\mathrm{P}<0.05)$.

The most frequently implicated nonmedicinal and medicinal compounds were reported in class T60 $(25.14 \%$, pesticides) and class T42 (8.55\%, antiepileptic, sedative-hypnotic and antiparkinsonism), respectively.

Among the overall non-medicinal groups, compounds in group T60 (25.14\%; pesticides) were responsible for the majority of intoxications, 
followed in order of frequency by substances in groups T54 (9.49\%; corrosive substances), T52 (5.92\%; organic solvents), T58 (2.88\%; carbon monoxide), T51 (1.85\%; alcohol), T63 (1.72\%; contact with venomous animals and plants), T55 (1.65\%; soaps and detergents), T65 (1.49\%; other and unspecified substances), T59 (0.57\%; other gases, fumes and vapors), T56 (0.55\%; metals), T61 (0.41\%; noxious substances eaten as seafood), and T62 $(0.21 \%$; other noxious substances eaten as food). Whereas, poisoning with substances in groups T53 $(0.0 \%$; halogen derivatives of aliphatic and aromatic hydrocarbons), T57 (0.0\%; other inorganic substances), and T64 (0.0\%; aflatoxin and other mycotoxin food contaminants) were not observed.

Poisoning with substances in groups $\mathrm{T} 51$ (3.79\%) and T63 (2.31\%) were mostly noticed among males, while females were mostly exposed to substances in groups T54 (10.27\%), T58 (3.33\%), and T60 (30.41\%). There were statistically significant variations among genders regarding the aforestated variables $(\mathrm{P}<0.05)$.

Among the overall medicinal groups, drugs in group T42 $(8.55 \%$, antiepileptic, sedative-hypnotic and antiparkinsonism) accounted for the first most commonly implicated group in acute poisoning, followed in order of frequency by medications in groups T39 (5.63\%; non-opioid analgesics, antipyretics and antirheumatics), T36 (4.69\%; systemic antibiotics), T40 (3.80\%; narcotics and psychodysleptics [hallucinogens]), T48 (2.74\%; agents primarily acting on muscles and the respiratory system), T38 (2.56\%; hormones and their synthetic substitutes and antagonists, not elsewhere classified), T44 (2.33\%; drugs primarily affecting the autonomic nervous system), T43 (2.17\%; psychotropic drugs, not elsewhere classified), T45 (2.13\%; primarily systemic and hematological agents, not elsewhere classified), T47 (1.92\%; agents primarily affecting the gastrointestinal system), T46 (1.56\%; agents primarily affecting the cardiovascular system), T37 (1.37\%; other systemic antiinfectives and antiparasitics), T49 $(0.85 \%$; topical agents primarily affecting skin and mucous membrane and by ophthalmological, otorhinolaryngological and dental drugs), and T50 (0.5\%; diuretics and other and unspecified substances). Whereas, poisoning with agents in group T41 (0.0\%; anesthetics and therapeutic gases) were not observed.

Poisoning with substances in groups T36 (7.58\%), T40 (8.41\%), T43 (2.86\%), T47 (2.42\%) were prevalent among males, while females were frequently exposed to substances in groups T39 $(6.86 \%)$ and T44 (3.1\%). There were statistically significant disparities among genders regarding the aforementioned variables $(\mathrm{P}<0.05)$.

Table (4) represents the distribution pattern of the most common involved medicinal and non-medicinal substances based on the ICD-10 classification. The most commonly involved causative agents among the overall non-medicinal and medicinal groups were organophosphate and carbamates compounds (20.24\%; T60-0) and benzodiazepine derivatives $(6.15 \%$; T42-4), respectively.

Among the overall non-medicinalrelated poisoning, organophosphate and carbamates $(20.24 \%$; T60-0) were the first most common causative agents implicated in acute poisoning, followed in order of frequency by alkali 
containing substances (4.67\%; T54-3), kerosene (3.93\%; T52-0), carbon monoxide due to incomplete combustion of carbonaceous materials $(2.26 \%$; T582), ethanol (1.33\%; T51-0), snake bites (1.24\%; T63-0), dishwashing and laundry (1.03\%; T55-1), mercury in thermometer $(0.43 \%$; T56-1), chlorine and hydrogen sulfide gases $(0.43 \%$; T59-4 and T59-6), ciguatera $(0.25 \%$; T61-0), and castor beans and datura seeds (0.21\%; T62-2) substances.

Causative agents in categories T51$0 \quad(2.75 \%), \quad$ T55-1 (1.48\%), T56-1 $(0.71 \%)$, and $\mathrm{T} 63-0 \quad(1.70 \%)$ were mostly involved in male poisoning, whilst, those in categories T52-0 $(4.82 \%)$ and $\mathrm{T} 60-0$ (24.69\%) were frequently implicated in female intoxication. There were statistically significant disproportions among genders concerning the aforementioned variables $(\mathrm{P}<0.05)$.

Among the overall medicinalrelated poisoning, benzodiazepines derivatives $(6.15 \%$; T42-4) constituted the first causative agents involved in acute poisoning, followed in order of frequency by acetaminophen and NSAIDs (4.57\%; T39-1 and T39-3), tramadol and cannabis $(3.11 \%$; T40-4 and T40-7), penicillin derivatives (2.40\%; T36-0), vitamins and iron (1.53\%; T45-2 and T45-4), theophylline (1.49\%; T48-6), oral hypoglycemic $(1.44 \%$; T38-3), tricyclic and tetracyclic antidepressants (1.42\%; T43-0), spasmolytic and $\beta$-adrenoreceptor antagonists (1.14\%; T44-3 and T44-5), anti-gastric-secretion and laxatives (0.94\%; T47-1 and T47-2), antiprotozoal and antiparasitics $(0.91 \%$; T37-3 and T37-8), cardiac-stimulant glycosides and calcium-channel blockers (0.87\%; T46-0 and T46-1), spironolactone containing diuretics
(0.71\%; T50-0), and local anti-infective and anti-inflammatory $(0.50 \%$; T49-0) preparations.

Causative agents in categories T383 (2.09\%), T40-4 and T40-7 (6.26\%), T43-0 (2.09\%), and T49-0 (0.77\%) were predominantly involved in male intoxication, whilst causative agent in category T42-4 $(6.82 \%)$ was commonly implicated in female poisoning. There were statistically significant disproportions among genders regarding the aforementioned variables $(\mathrm{P}<0.05)$.

Table (5) exhibits the distribution pattern of the clinical findings. The majority of cases were mildly intoxicated (77.13\%; Grade 1), admitted to the emergency room of the unit (70.31\%), hospitalized for $\leq 1$ day (73.19\%), and recovered completely without any sequelae $(97.71 \%)$. Meanwhile, the proportions of nonfatal sequelae $(1.44 \%)$ and mortality rate $(0.85 \%)$ were considerably low with organophosphates $(0.32 \%)$ were the predominant cause of death.

The patterns of clinical findings demonstrated that occurrence of moderate $(11.10 \%)$, severe $(9.73 \%)$, and fatal $(1.48 \%)$ manifestations, intensive care unit admission (8.24\%), hospital stay for $4(6.37 \%)$ and $5(3.57 \%)$ days, and nonfatal sequelae $(2.25 \%)$ as well as organophosphate $(0.55 \%)$, snake bites $(0.27 \%)$, and opioids $(0.27 \%)$ relateddeaths were more frequent recorded among male patients, whereas the occurrence of mild manifestation $(80.76 \%)$, emergency room admission $(72.22 \%)$, hospital stay for 2-day $(16.18 \%)$, and complete recovery $(98.75 \%)$ were more commonly reported among female patients. There were statistically significant differences between genders regarding the aforementioned variables $(\mathrm{P}<0.05)$. 
Table (6) describes the distribution pattern of the most common therapeutic interventions. The overall symptomatic patients received various initial therapies and according to the patients' requirements, one or more therapeutic interventions were applied. Utilizations of different decontamination procedures (67.34\%), especially internal decontamination $(64.75 \%)$ via single method $(50.64 \%)$, were the most frequently applied therapy and this was followed in frequency by the use of specific antidotes $(25.25 \%)$ and symptomatic treatment (18.02\%). However, emergent and supportive measures were used less frequently (9.13\%).

Among the overall cases, administration of activated charcoal (36.37\%, a local adsorbent antidote that effectively decreases the systemic absorption of most xenobiotics) was the most frequently used method, followed in order of frequency by performance of gastric lavage to remove stomach contents $(22.62 \%)$, induction of emesis with syrup of ipecac to evacuate the stomach (14.14\%), and oral administration of demulcents $(2.79 \%$; milk and/or egg white to sooth the gastric wall in corrosive poisoning). Administration of activated charcoal alone $(22.26 \%)$ was the most commonly applied single method, followed by gastric lavage (16.08\%), and syrup of ipecac (9.52\%). Additionally, concomitant use of gastric lavage and activated charcoal $(6.54 \%)$ was greater than combined use of syrup of ipecac and activated charcoal (4.62\%) or activated charcoal with cathartic sorbitol $(2.95 \%)$.

Utilizations

of internal decontamination procedures $(74.78 \%)$, especially activated charcoal (40.88\%) and gastric lavage (30.60\%), single method (59.07\%), particularly activated charcoal (25.16\%), gastric lavage (21.54\%), and demulcents (4.18\%), two methods $(15.71 \%)$, chiefly combined use of gastric lavage and activated charcoal $(9.07 \%)$ were more commonly applied among males, whereas the overall treatment with ipecac syrup $(15.91 \%)$, ipecac syrup alone $(10.46 \%)$, and combined ipecac syrup and activated charcoal (5.45\%) were considerably utilized among females. There were statistically significant differences between genders as regards the aforementioned variables $(\mathrm{P}<0.05)$.

Among the specific antidotes used, therapeutic administration of atropine alone $(13.08 \%)$ or together with obidoxime chloride $(3.91 \%)$ were the most commonly used specific antidotes, followed in order of frequency by utilization of sodium bicarbonate (2.15\%), N-acetylcysteine (1.85\%), naloxone $(1.19 \%)$, anticoagulant and vitamin $\mathrm{K}(0.98 \%)$, glucose $(0.80 \%)$, polyvalent snake antivenom $(0.43 \%)$, flumazenil $(0.37 \%)$, scorpion antivenom $(0.21 \%)$, and other antidotes $(0.27 \%)$.

Utilizations of all specific antidotes $(37.86 \%)$, atropine alone (21.76\%), atropine plus obidoxime chloride (5.55\%), naloxone $(2.20 \%)$, and polyvalent snake antivenom $(0.71 \%)$ as well as symptomatic treatment $(29.12 \%)$ and emergent and supportive measures $(15.88 \%)$ were more commonly required in treatment of males than females. There were statistically significant differences between genders in terms of the aforementioned variables $(\mathrm{P}<0.05)$. 
Table (1): The percentage of the distribution pattern of socio-epidemiological data regarding overall cases and both genders.

\begin{tabular}{|c|c|c|c|c|}
\hline \multirow[b]{2}{*}{ Variables } & Overall & Males & Females & \multirow[b]{2}{*}{ Z-value (P) } \\
\hline & $\begin{array}{c}\text { Number } \\
(\%)^{@}\end{array}$ & $\begin{array}{c}\text { Number } \\
(\%)^{\#}\end{array}$ & $\begin{array}{c}\text { Number } \\
(\%)^{\$}\end{array}$ & \\
\hline All studied cases & $\begin{array}{c}4372 \\
(100.00)\end{array}$ & $\begin{array}{c}1820 \\
(41.63)\end{array}$ & $\begin{array}{c}2552 \\
(58.37)\end{array}$ & $\begin{array}{c}15.7 \\
(0.0000)^{*}\end{array}$ \\
\hline \multicolumn{5}{|l|}{ Age (year) } \\
\hline$<18$ & $\begin{array}{c}2967 \\
(67.86)\end{array}$ & $\begin{array}{c}1379 \\
(75.77)\end{array}$ & $\begin{array}{c}1588 \\
(62.23)\end{array}$ & $\begin{array}{c}9.5 \\
(0.0000)^{*}\end{array}$ \\
\hline$<6$ & $\begin{array}{c}1700 \\
(38.88)\end{array}$ & $\begin{array}{c}1021 \\
(56.10)\end{array}$ & $679(26.61)$ & $\begin{array}{c}19.7 \\
(0.0000)^{*}\end{array}$ \\
\hline $7-12$ & $273(6.24)$ & $105(5.77)$ & $168(6.58)$ & $\begin{array}{c}1.1 \\
(0.2752)^{\mathrm{NS}}\end{array}$ \\
\hline $13-15$ & $541(12.37)$ & $154(8.46)$ & $387(15.16)$ & $\begin{array}{c}6.6 \\
(0.0001)^{*}\end{array}$ \\
\hline $16-<18$ & $453(10.36)$ & $99(5.44)$ & $354(13.87)$ & $9(0.0000)^{*}$ \\
\hline$\geq 18$ & $\begin{array}{c}1405 \\
(32.14)\end{array}$ & $441(24.23)$ & 964 (37.77) & $\begin{array}{c}9.5 \\
(0.0000)^{*}\end{array}$ \\
\hline 18-29 & 777 (17.77) & $272(14.95)$ & 505 (19.79) & $\begin{array}{c}4.1 \\
(0.0001)^{*}\end{array}$ \\
\hline 30-39 & 371 (8.49) & $105(5.77)$ & $266(10.42)$ & $\begin{array}{c}5.4 \\
(0.0001)^{*}\end{array}$ \\
\hline $40-49$ & $137(3.13)$ & $35(1.92)$ & $102(4.00)$ & $\begin{array}{c}3.9 \\
(0.0001)^{*}\end{array}$ \\
\hline$>50$ & $120(2.74)$ & $29(1.59)$ & $91(3.57)$ & $\begin{array}{c}3.9 \\
(0.0001)^{*}\end{array}$ \\
\hline \multicolumn{5}{|l|}{ Marital status } \\
\hline Married & $640(14.64)$ & $369(20.27)$ & $271(10.62)$ & $\begin{array}{c}8.9 \\
(0.0000)^{*}\end{array}$ \\
\hline Unmarried & $\begin{array}{c}3732 \\
(85.36)\end{array}$ & $\begin{array}{c}1451 \\
(79.73)\end{array}$ & $\begin{array}{c}2281 \\
(89.38)\end{array}$ & $\begin{array}{c}8.9 \\
(0.0000)^{*}\end{array}$ \\
\hline \multicolumn{5}{|l|}{ Residency } \\
\hline Rural & $\begin{array}{c}3064 \\
(70.08)\end{array}$ & $\begin{array}{c}1087 \\
(59.73)\end{array}$ & $\begin{array}{c}1977 \\
(77.47)\end{array}$ & $\begin{array}{c}12.6 \\
(0.0000)^{*}\end{array}$ \\
\hline Urban & $\begin{array}{c}1308 \\
(29.92)\end{array}$ & $733(40.27)$ & $575(22.53)$ & $\begin{array}{c}12.6 \\
(0.0000)^{*}\end{array}$ \\
\hline \multicolumn{5}{|l|}{ Income } \\
\hline Low & $\begin{array}{c}2963 \\
(67.77)\end{array}$ & $\begin{array}{c}1273 \\
(69.95)\end{array}$ & $\begin{array}{c}1690 \\
(66.22)\end{array}$ & $\begin{array}{c}2.6 \\
(0.0093)^{*}\end{array}$ \\
\hline Middle & $928(21.23)$ & 345 (18.96) & $583(22.84)$ & $3.1(0.002)^{*}$ \\
\hline High & $481(11.00)$ & $202(11.10)$ & $279(10.93)$ & $\begin{array}{c}0.2 \\
(0.8594)^{\mathrm{NS}}\end{array}$ \\
\hline \multicolumn{5}{|l|}{ Education $^{\phi}$} \\
\hline Low $^{\&}$ & 2136 & $681(37.42)$ & 1455 & 12.8 \\
\hline
\end{tabular}




\begin{tabular}{|c|c|c|c|c|}
\hline & $(48.86)$ & & $(57.01)$ & $(0.0000)^{*}$ \\
\hline Illiterate & 313 (7.16) & $99(5.44)$ & $214(8.39)$ & $\begin{array}{c}3.7 \\
(0.0002)^{*}\end{array}$ \\
\hline Primary school $^{\&}$ & $509(11.64)$ & $162(8.90)$ & $347(13.60)$ & $\begin{array}{c}4.8 \\
(0.0001)^{*}\end{array}$ \\
\hline Middle school $^{\&}$ & $654(14.96)$ & $199(10.93)$ & $455(17.83)$ & $\begin{array}{c}6.3 \\
(0.0001)^{*}\end{array}$ \\
\hline Secondary school ${ }^{\&}$ & $660(15.10)$ & $221(12.14)$ & $439(17.20)$ & $\begin{array}{c}4.6 \\
(0.0001)^{*}\end{array}$ \\
\hline High $^{\Psi}$ & $536(12.26)$ & $118(6.48)$ & $418(16.38)$ & $\begin{array}{c}9.8 \\
(0.0000)^{*}\end{array}$ \\
\hline \multicolumn{5}{|l|}{ Occupational status } \\
\hline All employed & $\begin{array}{c}2003 \\
(45.81)\end{array}$ & $806(44.29)$ & $\begin{array}{c}1197 \\
(46.90)\end{array}$ & $\begin{array}{c}1.7 \\
(0.0877)^{\mathrm{NS}}\end{array}$ \\
\hline Governmental & $132(3.02)$ & $56(3.08)$ & $76(2.98)$ & $\begin{array}{c}0.2 \\
(0.849)^{\mathrm{NS}}\end{array}$ \\
\hline Non-governmental $^{\Omega}$ & $231(5.28)$ & $79(4.34)$ & $152(5.96)$ & $\begin{array}{c}2.4 \\
(0.0183)^{*}\end{array}$ \\
\hline Household workers & $\begin{array}{c}1002 \\
(22.92) \\
\end{array}$ & $341(18.74)$ & $661(25.90)$ & $\begin{array}{c}5.6 \\
(0.0001)^{*}\end{array}$ \\
\hline Manual workers & $295(6.75)$ & $110(6.04)$ & $185(7.25)$ & $\begin{array}{c}1.6 \\
(0.1159)^{\mathrm{NS}}\end{array}$ \\
\hline Professional workers & $159(3.64)$ & $114(6.26)$ & $45(1.76)$ & $\begin{array}{c}7.8 \\
(0.0001)^{*}\end{array}$ \\
\hline Agricultural workers & $141(3.23)$ & $91(5.00)$ & $50(1.96)$ & $\begin{array}{c}5.6 \\
(0.0001)^{*}\end{array}$ \\
\hline Other jobs & $43(0.98)$ & $15(0.82)$ & $28(1.10)$ & $\begin{array}{c}0.9 \\
(0.355)^{\mathrm{NS}}\end{array}$ \\
\hline All unemployed (Jobless) & $\begin{array}{c}2369 \\
(54.19) \\
\end{array}$ & $\begin{array}{c}1014 \\
(55.71) \\
\end{array}$ & $\begin{array}{c}1355 \\
(53.10) \\
\end{array}$ & $\begin{array}{c}1.7 \\
(0.0877)^{\mathrm{NS}}\end{array}$ \\
\hline \multicolumn{5}{|l|}{$\begin{array}{c}\text { Past history of health } \\
\text { status }\end{array}$} \\
\hline $\begin{array}{c}\text { Mental and physical } \\
\text { disability }\end{array}$ & $236(5.40)$ & $139(7.64)$ & $97(3.80)$ & $\begin{array}{c}5.5 \\
(0.0001)^{*}\end{array}$ \\
\hline $\begin{array}{c}\text { Other concomitant } \\
\text { diseases }^{\odot}\end{array}$ & $750(17.15)$ & $258(14.18)$ & $492(19.28)$ & $\begin{array}{c}4.4 \\
(0.0001)^{*}\end{array}$ \\
\hline Normal & $\begin{array}{c}3386 \\
(77.45)\end{array}$ & $\begin{array}{c}1423 \\
(78.19)\end{array}$ & $\begin{array}{c}1963 \\
(76.92)\end{array}$ & $\begin{array}{c}1 \\
(0.3219)^{\mathrm{NS}}\end{array}$ \\
\hline
\end{tabular}

$@=$ Percentage in relation to $4372(100 \%)$ cases; \#=Percentage in relation to 1820 $(100 \%)$ cases; $\$=$ Percentage in relation to $2552(100 \%)$ cases; $P=$ Probability; $*=$ Significant difference at the 0.05 level; NS=No significant difference; <=less than; $\geq=$ Greater than or equal to; $>=$ more than; $\phi=$ Pre-school children were excluded; $\&=$ Illiterates and those registered in or graduated from primary, middle, and secondary schools; $\Psi=$ Those registered in or graduated from university; $\Omega=$ =Include jobholders, private sector, self-employed, or other; $\odot=$ =Such as diabetes mellitus, hypertension, cardiovascular, pulmonary, hepato-renal, or other diseases. 
Table (2): The percentage of distribution pattern of toxicological data regarding overall cases and both genders.

\begin{tabular}{|c|c|c|c|c|}
\hline Variables & $\begin{array}{c}\text { Overall } \\
\text { Number }\end{array}$ & $\begin{array}{c}\text { Males } \\
\text { Number }\end{array}$ & $\begin{array}{l}\text { Females } \\
\text { Number }\end{array}$ & $\begin{array}{c}\text { Z-value } \\
\text { (P) }\end{array}$ \\
\hline \multicolumn{5}{|c|}{$\begin{array}{l}\text { The location of poisoning } \\
\text { exposure }\end{array}$} \\
\hline Home & $\begin{array}{c}4023 \\
(92.02)\end{array}$ & $\begin{array}{c}1563 \\
(85.88)\end{array}$ & $\begin{array}{c}2460 \\
(96.39)\end{array}$ & $\begin{array}{c}12.6 \\
(0.0000)^{*}\end{array}$ \\
\hline Other Places & $349(7.98)$ & $\begin{array}{c}257 \\
(14.12)\end{array}$ & $92(3.61)$ & $\begin{array}{c}12.6 \\
(0.0001)^{*}\end{array}$ \\
\hline \multicolumn{5}{|c|}{ Pre-hospitalization (Hours) } \\
\hline$\leq 1$-hour & $517(11.83)$ & $\begin{array}{c}221 \\
(12.14)\end{array}$ & $\begin{array}{c}296 \\
(11.60)\end{array}$ & $\begin{array}{c}0.5 \\
(0.5857)^{\mathrm{NS}}\end{array}$ \\
\hline 2-hour & $751(17.18)$ & $\begin{array}{c}350 \\
(19.23)\end{array}$ & $\begin{array}{c}401 \\
(15.71)\end{array}$ & $\begin{array}{c}3 \\
(0.0024)^{*}\end{array}$ \\
\hline 3-hour & $\begin{array}{c}1860 \\
(42.54)\end{array}$ & $\begin{array}{c}800 \\
(43.96)\end{array}$ & $\begin{array}{c}1060 \\
(41.54)\end{array}$ & $\begin{array}{c}1.6 \\
(0.1106)^{\mathrm{NS}}\end{array}$ \\
\hline 4-hour & 994 (22.74) & $\begin{array}{c}341 \\
(18.74)\end{array}$ & $\begin{array}{c}653 \\
(25.59)\end{array}$ & $\begin{array}{c}5.3 \\
(0.0001)^{*}\end{array}$ \\
\hline$\geq 5$-hour & $250(5.72)$ & $\begin{array}{c}108 \\
(5.93)\end{array}$ & $\begin{array}{c}142 \\
(5.56)\end{array}$ & $\begin{array}{c}0.5 \\
(0.6034)^{\mathrm{NS}}\end{array}$ \\
\hline \multicolumn{5}{|c|}{ Admission shifts } \\
\hline Morning & $670(15.32)$ & $\begin{array}{c}188 \\
(10.33)\end{array}$ & $\begin{array}{c}482 \\
(18.89)\end{array}$ & $\begin{array}{c}7.7 \\
(0.0001)^{*}\end{array}$ \\
\hline Afternoon & $2119(48.47)$ & $\begin{array}{c}564 \\
(30.99)\end{array}$ & $\begin{array}{c}1555 \\
(60.93)\end{array}$ & $\begin{array}{c}19.5 \\
(0.0000)^{*}\end{array}$ \\
\hline Evening & $1139(26.05)$ & $\begin{array}{c}787 \\
(43.24)\end{array}$ & $\begin{array}{c}352 \\
(13.79)\end{array}$ & $\begin{array}{c}21.9 \\
(0.0000)^{*}\end{array}$ \\
\hline Night & $444(10.16)$ & $\begin{array}{c}281 \\
(15.44)\end{array}$ & $\begin{array}{c}163 \\
(6.39)\end{array}$ & $\begin{array}{c}9.8 \\
(0.0000)^{*}\end{array}$ \\
\hline \multicolumn{5}{|c|}{ Seasonal variations } \\
\hline Summer & $\begin{array}{c}1538 \\
(35.18)\end{array}$ & $\begin{array}{c}486 \\
(26.70)\end{array}$ & $\begin{array}{c}1052 \\
(41.22)\end{array}$ & $\begin{array}{c}9.9 \\
(0.0000)^{*}\end{array}$ \\
\hline Spring & $\begin{array}{c}1375 \\
(31.45)\end{array}$ & $\begin{array}{c}900 \\
(49.45)\end{array}$ & $\begin{array}{c}475 \\
(18.61)\end{array}$ & $\begin{array}{c}21.6 \\
(0.0000)^{*}\end{array}$ \\
\hline Autumn & $888(20.31)$ & $\begin{array}{c}239 \\
(13.13)\end{array}$ & $\begin{array}{c}649 \\
(25.43)\end{array}$ & $\begin{array}{c}10 \\
(0.0000)^{*}\end{array}$ \\
\hline Winter & $571(13.06)$ & $\begin{array}{c}195 \\
(10.71)\end{array}$ & $\begin{array}{c}376 \\
(14.73)\end{array}$ & $\begin{array}{c}3.9 \\
(0.0001)^{*}\end{array}$ \\
\hline \multicolumn{5}{|c|}{ Routes of Exposure } \\
\hline Oral & $\begin{array}{c}3714 \\
(84.95)\end{array}$ & $\begin{array}{c}1532 \\
(84.18)\end{array}$ & $\begin{array}{c}2182 \\
(85.50)\end{array}$ & $\begin{array}{c}1.2 \\
(0.2289)^{\mathrm{NS}}\end{array}$ \\
\hline Pulmonary & $410(9.38)$ & $\begin{array}{c}157 \\
(8.63)\end{array}$ & $\begin{array}{c}253 \\
(9.91)\end{array}$ & $\begin{array}{c}1.4 \\
(0.1524)^{\mathrm{NS}}\end{array}$ \\
\hline
\end{tabular}




\begin{tabular}{|c|c|c|c|c|}
\hline Skin puncture & $121(2.77)$ & $85(4.67)$ & $36(1.41)$ & $\begin{array}{c}6.5 \\
(0.0001)^{*}\end{array}$ \\
\hline Dermal contact & $103(2.36)$ & $25(1.37)$ & $78(3.06)$ & $\begin{array}{c}3.6 \\
(0.0003)^{*}\end{array}$ \\
\hline Snorting & & & & 4.5 \\
& $24(0.55)$ & $21(1.15)$ & $3(0.12)$ & $(0.0001)^{*}$ \\
\hline Manner of exposure & & & & \\
\hline Intentional & 1933 & 532 & 1401 & 16.8 \\
& $(44.21)$ & $(29.23)$ & $(54.90)$ & $(0.0000)^{*}$ \\
\hline Unintentional & 2439 & 1288 & 1151 & 16.8 \\
& $(55.79)$ & $(70.77)$ & $(45.10)$ & $(0.0000)^{*}$ \\
\hline The number of cases/number of & & & & \\
substances & & & & \\
\hline $\begin{array}{c}\text { All cases exposed to single } \\
\text { agents }\end{array}$ & 4052 & 1685 & 2367 & 0.2 \\
\hline All cases exposed to multiple & $(92.68)$ & $(92.58)$ & $(92.75)$ & $(0.8315)^{\mathrm{NS}}$ \\
agents & $320(7.32)$ & $(7.42)$ & $(7.25)$ & $(0.8315)^{\mathrm{NS}}$ \\
\hline All number of implicated & 4856 & 2049 & 2807 & 0.3 \\
poisonous agents & $(111.07)$ & $(112.58)$ & $(109.99)$ & $(0.7874)^{\mathrm{NS}}$ \\
\hline Medicinal & 2334 & 1081 & 1253 & 6.7 \\
& $(53.39)$ & $(59.40)$ & $(49.10)$ & $(0.0001)^{*}$ \\
\hline Non-Medicinal & 2522 & 968 & 1554 & 5.1 \\
& $(57.69)$ & $(53.19)$ & $(60.89)$ & $(0.0001)^{*}$ \\
\hline All involved multiple & & 364 & 440 & 2.3 \\
substances & $804(18.39)$ & $(20.00)$ & $(17.24)$ & $(0.0202)^{*}$ \\
\hline Medicinal & & 212 & 338 & 1.6 \\
& $550(12.58)$ & $(11.65)$ & $(13.24)$ & $(0.1181)^{\mathrm{NS}}$ \\
\hline Non-Medicinal & $254(5.81)$ & $(8.35)$ & $(4.00)$ & $(0.0001)^{*}$ \\
\hline
\end{tabular}

$@=$ Percentage in relation to $4372(100 \%)$ cases; \#=Percentage in relation to 1820

$(\mathbf{1 0 0 \%})$ cases; $\$=$ Percentage in relation to 2552 (100\%) cases; $P=$ Probability; *=Significant difference at the 0.05 level; NS=No significant difference; $\leq=$ less than or equal to; $\geq=$ Greater than or equal to; $\Omega=$ None homicidal cases recorded; $\&=$ All single + multiple involved substances divided by the total number of poisoned cases (4856/4372). 
Table (3): The percentage of distribution pattern of implicated medicinal and nonmedicinal groups regarding overall cases and both genders based on the ICD-10 classification.

\begin{tabular}{|c|c|c|c|c|c|}
\hline Codes & Categories & Overall & Male & Female & \multirow[b]{2}{*}{$\begin{array}{l}\text { Z-value } \\
\text { (P) }\end{array}$} \\
\hline & & $\begin{array}{c}\text { Number } \\
(\%)^{@}\end{array}$ & $\begin{array}{c}\text { Number } \\
(\%)^{\#}\end{array}$ & $\begin{array}{c}\text { Number } \\
(\%)^{\$}\end{array}$ & \\
\hline & Medicinal & & & & \\
\hline T36 & Systemic antibiotics & $\begin{array}{l}205 \\
(4.69)\end{array}$ & $\begin{array}{l}138 \\
(7.58)\end{array}$ & $\begin{array}{l}67 \\
(2.63)\end{array}$ & $\begin{array}{l}7.6 \\
(0.0001)^{*}\end{array}$ \\
\hline $\mathrm{T} 37$ & $\begin{array}{l}\text { Other systemic anti-infectives and } \\
\text { antiparasitics }\end{array}$ & $\begin{array}{l}60 \\
(1.37)\end{array}$ & $\begin{array}{l}22 \\
(1.21)\end{array}$ & $\begin{array}{l}38 \\
(1.49)\end{array}$ & $\begin{array}{l}0.8 \\
(0.433)^{\mathrm{NS}}\end{array}$ \\
\hline T38 & $\begin{array}{l}\text { Hormones and their synthetic } \\
\text { substitutes and antagonists, NEC }\end{array}$ & $\begin{array}{l}112 \\
(2.56)\end{array}$ & $\begin{array}{l}45 \\
(2.47)\end{array}$ & $\begin{array}{l}67 \\
(2.63)\end{array}$ & $\begin{array}{l}0.3 \\
(0.7414)^{\mathrm{NS}}\end{array}$ \\
\hline T39 & $\begin{array}{l}\text { Nonopioid analgesics, antipyretics } \\
\text { and antirheumatics }\end{array}$ & $\begin{array}{l}246 \\
(5.63)\end{array}$ & $\begin{array}{l}71 \\
(3.90) \\
\end{array}$ & $\begin{array}{l}175 \\
(6.86)\end{array}$ & $\begin{array}{l}4.2 \\
(0.0001)^{*}\end{array}$ \\
\hline $\mathrm{T} 40$ & $\begin{array}{l}\text { Narcotics and psychodysleptics } \\
\text { [hallucinogens] }\end{array}$ & $\begin{array}{l}166 \\
(3.80)\end{array}$ & $\begin{array}{l}153 \\
(8.41)\end{array}$ & $\begin{array}{l}13 \\
(0.51)\end{array}$ & $\begin{array}{l}13.5 \\
(0.0000)^{*}\end{array}$ \\
\hline T41 & Anesthetics and therapeutic gases & \multicolumn{4}{|c|}{ Not observed } \\
\hline T42 & $\begin{array}{l}\text { Antiepileptic, sedative-hypnotic and } \\
\text { antiparkinsonism drugs }\end{array}$ & $\begin{array}{l}374 \\
(8.55)\end{array}$ & $\begin{array}{l}173 \\
(9.51)\end{array}$ & $\begin{array}{l}201 \\
(7.88)\end{array}$ & $\begin{array}{l}1.9 \\
(0.0575)^{\mathrm{NS}}\end{array}$ \\
\hline $\mathrm{T} 43$ & Psychotropic drugs, NEC & $\begin{array}{l}95 \\
(2.17)\end{array}$ & $\begin{array}{l}52 \\
(2.86)\end{array}$ & $\begin{array}{l}43 \\
(1.68)\end{array}$ & $\begin{array}{l}2.6 \\
(0.0083)^{*}\end{array}$ \\
\hline T44 & $\begin{array}{l}\text { Drugs } \\
\text { autono }\end{array}$ & $\begin{array}{l}102 \\
(2.33)\end{array}$ & $\begin{array}{l}23 \\
(1.26)\end{array}$ & $\begin{array}{l}79 \\
(3.10)\end{array}$ & $\begin{array}{l}4 \\
(0.0001)^{*}\end{array}$ \\
\hline T45 & $\begin{array}{l}\text { Primarily systemic and } \\
\text { hematological agents, NEC }\end{array}$ & $\begin{array}{l}93 \\
(2.13) \\
\end{array}$ & $\begin{array}{l}36 \\
(1.98)\end{array}$ & $\begin{array}{l}57 \\
(2.23) \\
\end{array}$ & $\begin{array}{l}0.6 \\
(0.5721)^{\mathrm{NS}}\end{array}$ \\
\hline T46 & $\begin{array}{l}\text { Agents primarily affecting the } \\
\text { cardiovascular system }\end{array}$ & $\begin{array}{l}68 \\
(1.56)\end{array}$ & $\begin{array}{l}27 \\
(1.48)\end{array}$ & $\begin{array}{l}41 \\
(1.61)\end{array}$ & $\begin{array}{l}0.3 \\
(0.7321)^{\mathrm{NS}}\end{array}$ \\
\hline T47 & $\begin{array}{l}\text { Agents primarily } \\
\text { gastrointestinal sy }\end{array}$ & $\begin{array}{l}84 \\
(1.92)\end{array}$ & $\begin{array}{l}44 \\
(2.42)\end{array}$ & $\begin{array}{l}40 \\
(1.57)\end{array}$ & $\begin{array}{l}2 \\
(0.0437)^{*}\end{array}$ \\
\hline $\mathrm{T} 48$ & $\begin{array}{l}\text { Agents primarily acting on muscles } \\
\text { and the respiratory system }\end{array}$ & $\begin{array}{l}120 \\
(2.74)\end{array}$ & $\begin{array}{l}59 \\
(3.24)\end{array}$ & $\begin{array}{l}61 \\
(2.39)\end{array}$ & $\begin{array}{l}1.7 \\
(0.0899)^{\mathrm{NS}}\end{array}$ \\
\hline T49 & Topical agents & $\begin{array}{l}37 \\
(0.85)\end{array}$ & $\begin{array}{l}17 \\
(0.93)\end{array}$ & $\begin{array}{l}20 \\
(0.78)\end{array}$ & $\begin{array}{l}0.5 \\
(0.5927)^{\mathrm{NS}}\end{array}$ \\
\hline \multirow[t]{5}{*}{ T50 } & $\begin{array}{l}\text { Diuretics and other and unspecified } \\
\text { substances }\end{array}$ & $\begin{array}{l}22 \\
(0.50)\end{array}$ & $9(0.49)$ & $\begin{array}{l}13 \\
(0.51)\end{array}$ & $\begin{array}{l}0.1 \\
(0.9265)^{\mathrm{NS}}\end{array}$ \\
\hline & $\begin{array}{l}\text { Total cases exposed to single } \\
\text { medicinal substance }\end{array}$ & $\begin{array}{l}1784 \\
(40.81)\end{array}$ & $\begin{array}{l}869 \\
(47.75)\end{array}$ & $\begin{array}{l}915 \\
(35.85)\end{array}$ & $\begin{array}{l}7.9 \\
(0.0001)^{*}\end{array}$ \\
\hline & $\begin{array}{l}\text { Total cases exposed to multiple } \\
\text { medicinal substances }\end{array}$ & $\begin{array}{l}201 \\
(4.60)\end{array}$ & $\begin{array}{l}70 \\
(3.85)\end{array}$ & $\begin{array}{l}131 \\
(5.13)\end{array}$ & $\begin{array}{l}2 \\
(0.0464)^{*}\end{array}$ \\
\hline & $\begin{array}{l}\text { Total cases exposed to single and } \\
\text { multiple medicinal substances }\end{array}$ & $\begin{array}{l}1985 \\
(45.40)\end{array}$ & $\begin{array}{l}939 \\
(51.59)\end{array}$ & $\begin{array}{l}1046 \\
(40.99)\end{array}$ & $\begin{array}{l}6.9 \\
(0.0001)^{*}\end{array}$ \\
\hline & $\mathrm{N}$ & & & & \\
\hline T51 & Alcohol & 81 & 69 & 12 & 8 \\
\hline
\end{tabular}




\begin{tabular}{|c|c|c|c|c|c|}
\hline & & $(1.85)$ & $(3.79)$ & $(0.47)$ & $(0.0001)^{*}$ \\
\hline T52 & Organic solvents & $\begin{array}{l}259 \\
(5.92)\end{array}$ & $\begin{array}{l}93 \\
(5.11)\end{array}$ & $\begin{array}{l}166 \\
(6.50)\end{array}$ & $\begin{array}{l}1.9 \\
(0.0549)^{\mathrm{NS}}\end{array}$ \\
\hline $\mathrm{T} 53$ & $\begin{array}{l}\text { Halogen derivatives of aliphatic and } \\
\text { aromatic hydrocarbons }\end{array}$ & \multicolumn{4}{|c|}{ Not observed } \\
\hline T54 & Corrosive substances & $\begin{array}{l}415 \\
(9.49)\end{array}$ & $\begin{array}{l}153 \\
(8.41)\end{array}$ & $\begin{array}{l}262 \\
(10.27)\end{array}$ & $\begin{array}{l}2.1 \\
(0.0386)^{*}\end{array}$ \\
\hline $\mathrm{T} 55$ & Soaps and detergents & $\begin{array}{l}72 \\
(1.65)\end{array}$ & $\begin{array}{l}25 \\
(1.37)\end{array}$ & $\begin{array}{l}47 \\
(1.84)\end{array}$ & $\begin{array}{l}1.2 \\
(0.2284)^{\mathrm{NS}}\end{array}$ \\
\hline T56 & Metals & $\begin{array}{l}24 \\
(0.55)\end{array}$ & $\begin{array}{l}14 \\
(0.77)\end{array}$ & $\begin{array}{l}10 \\
(0.39)\end{array}$ & $\begin{array}{l}1.7 \\
(0.0935)^{\mathrm{NS}}\end{array}$ \\
\hline T57 & Other inorganic substances & \multicolumn{4}{|c|}{ Not observed } \\
\hline T58 & Carbon monoxide & $\begin{array}{l}126 \\
(2.88)\end{array}$ & $\begin{array}{l}41 \\
(2.25)\end{array}$ & $\begin{array}{l}85 \\
(3.33)\end{array}$ & $\begin{array}{l}2.1 \\
(0.0353)^{*}\end{array}$ \\
\hline T59 & Other gases, fumes and vapors & $\begin{array}{l}25 \\
(0.57)\end{array}$ & $\begin{array}{l}14 \\
(0.77)\end{array}$ & $\begin{array}{l}11 \\
(0.43)\end{array}$ & $\begin{array}{l}1.5 \\
(0.1415)^{\mathrm{NS}}\end{array}$ \\
\hline T60 & Pesticides & $\begin{array}{l}1099 \\
(25.14)\end{array}$ & $\begin{array}{l}323 \\
(17.75)\end{array}$ & $\begin{array}{l}776 \\
(30.41)\end{array}$ & $\begin{array}{l}9.5 \\
(0.0000)^{*}\end{array}$ \\
\hline T61 & Noxious substances eaten as seafood & $\begin{array}{l}18 \\
(0.41)\end{array}$ & $\begin{array}{l}11 \\
(0.60)\end{array}$ & $7(0.27)$ & $\begin{array}{l}1.7 \\
(0.0913)^{\mathrm{NS}}\end{array}$ \\
\hline T62 & $\begin{array}{l}\text { Other noxious substances eaten as } \\
\text { food }\end{array}$ & $9(0.21)$ & $4(0.22)$ & $5(0.20)$ & $\begin{array}{l}0.1 \\
(0.8863)^{\mathrm{NS}}\end{array}$ \\
\hline T63 & $\begin{array}{l}\text { Contact with venomous animals and } \\
\text { plants }\end{array}$ & $\begin{array}{l}75 \\
(1.72)\end{array}$ & $\begin{array}{l}42 \\
(2.31)\end{array}$ & $\begin{array}{l}33 \\
(1.29)\end{array}$ & $\begin{array}{l}2.6 \\
(0.0104)^{*}\end{array}$ \\
\hline T64 & $\begin{array}{l}\text { Aflatoxin and other mycotoxin food } \\
\text { contaminants }\end{array}$ & \multicolumn{4}{|c|}{ Not observed } \\
\hline \multirow[t]{4}{*}{ T65 } & Other and unspecified substances & $\begin{array}{l}65 \\
(1.49)\end{array}$ & $\begin{array}{l}27 \\
(1.48)\end{array}$ & $\begin{array}{l}38 \\
(1.49)\end{array}$ & $\begin{array}{l}0.03 \\
(0.9785)^{\mathrm{NS}}\end{array}$ \\
\hline & $\begin{array}{l}\text { Total cases exposed to single non- } \\
\text { medicinal substance }\end{array}$ & $\begin{array}{l}2268 \\
(51.88)\end{array}$ & $\begin{array}{l}816 \\
(44.84)\end{array}$ & $\begin{array}{l}1452 \\
(56.90)\end{array}$ & $\begin{array}{l}7.9 \\
(0.0001)^{*}\end{array}$ \\
\hline & $\begin{array}{l}\text { Total cases exposed to multiple non- } \\
\text { medicinal substances }\end{array}$ & $\begin{array}{l}119 \\
(2.72)\end{array}$ & $\begin{array}{l}65 \\
(3.57)\end{array}$ & $\begin{array}{l}54 \\
(2.12)\end{array}$ & $\begin{array}{l}2.9 \\
(0.0037)^{*}\end{array}$ \\
\hline & $\begin{array}{l}\text { Total cases exposed to single and } \\
\text { multiple non-medicinal substances }\end{array}$ & $\begin{array}{l}2387 \\
(54.60)\end{array}$ & $\begin{array}{l}881 \\
(48.41)\end{array}$ & $\begin{array}{l}1506 \\
(59.01)\end{array}$ & $\begin{array}{l}6.9 \\
(0.0001)^{*}\end{array}$ \\
\hline
\end{tabular}

$@=$ Percentage in relation to $4372(100 \%)$ cases; \#=Percentage in relation to $1820(100 \%)$ cases; $\$=$ Percentage in relation to $2552(100 \%)$ cases; $P=P r o b a b i l i t y ; *=$ Significant difference at the 0.05 level; $N S=$ No significant difference; $N E C=$ Not elsewhere classified; T49=Skin and mucous membrane, ophthalmological, otorhinorlaryngological and dental primarily affecting drugs. 
Table (4): The percentage distribution pattern of the most common involved medicinal and non-medicinal causative agents regarding overall cases and both genders based on the ICD-10 classification.

\begin{tabular}{|c|c|c|c|c|c|}
\hline \multirow[b]{2}{*}{ Codes } & \multirow[b]{2}{*}{ Categories } & Overall & Male & Female & \multirow[b]{2}{*}{$\begin{array}{l}\text { Z-value } \\
\quad(\mathrm{P})\end{array}$} \\
\hline & & $\begin{array}{l}\text { Number } \\
(\%)^{@}\end{array}$ & $\begin{array}{c}\text { Number } \\
(\%)^{\#}\end{array}$ & $\begin{array}{c}\text { Number } \\
(\%)^{\$}\end{array}$ & \\
\hline & Medicinal substances & & & & \\
\hline T36-0 & Penicillin derivatives & $\begin{array}{l}105 \\
(2.40)\end{array}$ & $\begin{array}{l}46 \\
(2.53)\end{array}$ & $\begin{array}{l}59 \\
(2.31)\end{array}$ & $\begin{array}{l}0.5 \\
(0.6395)^{\mathrm{NS}}\end{array}$ \\
\hline $\begin{array}{l}\text { T37-3 } \\
\text { T37-8 }\end{array}$ & $\begin{array}{l}\text { Antiprotozoal } \\
\text { Antiparasitics }\end{array}$ & $\begin{array}{l}40 \\
(0.91)\end{array}$ & $\begin{array}{l}15 \\
(0.82)\end{array}$ & $\begin{array}{l}25 \\
(0.98)\end{array}$ & $\begin{array}{l}0.5 \\
(0.5836)^{\mathrm{NS}}\end{array}$ \\
\hline T38-3 & Oral hypoglycemic & $\begin{array}{l}63 \\
(1.44)\end{array}$ & $\begin{array}{l}38 \\
(2.09)\end{array}$ & $\begin{array}{l}25 \\
(0.98)\end{array}$ & $\begin{array}{l}3 \\
(0.0024)^{*}\end{array}$ \\
\hline $\begin{array}{l}\text { T39-1 } \\
\text { T39-3 }\end{array}$ & $\begin{array}{l}\text { Acetaminophen } \\
\text { NSAIDs }\end{array}$ & $\begin{array}{l}200 \\
(4.57)\end{array}$ & $\begin{array}{l}91 \\
(5.00)\end{array}$ & $\begin{array}{l}109 \\
(4.27)\end{array}$ & $\begin{array}{l}1.1 \\
(0.2547)^{\mathrm{NS}}\end{array}$ \\
\hline $\begin{array}{l}\text { T40-4 } \\
\text { T40-7 }\end{array}$ & $\begin{array}{l}\text { Tramadol } \\
\text { Cannabis }\end{array}$ & $\begin{array}{l}136 \\
(3.11)\end{array}$ & $\begin{array}{l}114 \\
(6.26)\end{array}$ & $\begin{array}{l}22 \\
(0.86)\end{array}$ & $\begin{array}{l}10.1 \\
(0.0000)^{*}\end{array}$ \\
\hline T42-4 & Benzodiazepine derivatives & $\begin{array}{l}269 \\
(6.15)\end{array}$ & $\begin{array}{l}95 \\
(5.22)\end{array}$ & $\begin{array}{l}174 \\
(6.82)\end{array}$ & $2.2(0.03)^{*}$ \\
\hline T43-0 & $\begin{array}{l}\text { Tricyclic and Tetracyclic } \\
\text { antidepressants }\end{array}$ & $\begin{array}{l}62 \\
(1.42)\end{array}$ & $\begin{array}{l}38 \\
(2.09)\end{array}$ & $\begin{array}{l}24 \\
(0.94)\end{array}$ & $\begin{array}{l}3.2 \\
(0.0015)^{*}\end{array}$ \\
\hline $\begin{array}{l}\mathrm{T} 44-3 \\
\mathrm{~T} 44-5\end{array}$ & $\begin{array}{l}\text { Spasmolytics } \\
\text { Beta-adrenoreceptor antagonists }\end{array}$ & $\begin{array}{l}50 \\
(1.14)\end{array}$ & $\begin{array}{l}21 \\
(1.15)\end{array}$ & $\begin{array}{l}29 \\
(1.14)\end{array}$ & $\begin{array}{l}0.03 \\
(0.9756)^{\mathrm{NS}}\end{array}$ \\
\hline $\begin{array}{l}\mathrm{T} 45-2 \\
\mathrm{~T} 45-4\end{array}$ & $\begin{array}{l}\text { Vitamins } \\
\text { Iron compounds }\end{array}$ & $\begin{array}{l}67 \\
(1.53)\end{array}$ & $\begin{array}{l}35 \\
(1.92)\end{array}$ & $\begin{array}{l}32 \\
(1.25)\end{array}$ & $\begin{array}{l}1.8 \\
(0.0751)^{\mathrm{NS}}\end{array}$ \\
\hline $\begin{array}{l}\text { T46-0 } \\
\text { T46-1 }\end{array}$ & $\begin{array}{l}\text { Cardiac-stimulant glycosides } \\
\text { Calcium-channel blockers }\end{array}$ & $\begin{array}{l}38 \\
(0.87)\end{array}$ & $\begin{array}{l}15 \\
(0.82)\end{array}$ & $\begin{array}{l}23 \\
(0.90)\end{array}$ & $\begin{array}{l}0.3 \\
(0.7785)^{\mathrm{NS}}\end{array}$ \\
\hline $\begin{array}{l}\mathrm{T} 47-1 \\
\mathrm{~T} 47-2\end{array}$ & $\begin{array}{l}\text { Anti-gastric-secretion } \\
\text { Laxatives }\end{array}$ & $\begin{array}{l}41 \\
(0.94)\end{array}$ & $\begin{array}{l}23 \\
(1.26)\end{array}$ & $\begin{array}{l}18 \\
(0.71)\end{array}$ & $\begin{array}{l}1.9 \\
(0.0631)^{\mathrm{NS}}\end{array}$ \\
\hline T48-6 & Theophylline & $\begin{array}{l}65 \\
(1.49)\end{array}$ & $\begin{array}{l}28 \\
(1.54)\end{array}$ & $\begin{array}{l}37 \\
(1.45)\end{array}$ & $\begin{array}{l}0.2 \\
(0.8085)^{\mathrm{NS}}\end{array}$ \\
\hline T49-0 & $\begin{array}{l}\text { Local anti-infective and anti- } \\
\text { inflammatory drugs }\end{array}$ & $\begin{array}{l}22 \\
(0.50)\end{array}$ & $\begin{array}{l}14 \\
(0.77)\end{array}$ & $8(0.31)$ & $\begin{array}{l}2.1 \\
(0.0338)^{*}\end{array}$ \\
\hline T50-0 & $\begin{array}{l}\text { Spironolactone containing } \\
\text { diuretics }\end{array}$ & $\begin{array}{l}31 \\
(0.71)\end{array}$ & $\begin{array}{l}13 \\
(0.71)\end{array}$ & $\begin{array}{l}18 \\
(0.71)\end{array}$ & $0(1)^{\mathrm{NS}}$ \\
\hline & Non-medicinal substances & & & & \\
\hline T51-0 & Ethanol & $\begin{array}{l}58 \\
(1.33)\end{array}$ & $\begin{array}{l}50 \\
(2.75)\end{array}$ & $8(0.31)$ & $\begin{array}{l}7 \\
(0.0001)^{*}\end{array}$ \\
\hline T52-0 & Kerosene & $\begin{array}{l}172 \\
(3.93)\end{array}$ & $\begin{array}{l}49 \\
(2.69)\end{array}$ & $\begin{array}{l}123 \\
(4.82)\end{array}$ & $\begin{array}{l}3.6 \\
(0.0004)^{*}\end{array}$ \\
\hline T54-3 & Alkali containing substances & $\begin{array}{l}204 \\
(4.67)\end{array}$ & $\begin{array}{l}78 \\
(4.29)\end{array}$ & $\begin{array}{l}126 \\
(4.94)\end{array}$ & $\begin{array}{l}1 \\
(0.3153)^{\mathrm{NS}}\end{array}$ \\
\hline T55-1 & $\begin{array}{l}\text { Dishwashing and laundry } \\
\text { detergents }\end{array}$ & $\begin{array}{l}45 \\
(1.03)\end{array}$ & $\begin{array}{l}27 \\
(1.48)\end{array}$ & $\begin{array}{l}18 \\
(0.71)\end{array}$ & $\begin{array}{l}2.5 \\
(0.013)^{*}\end{array}$ \\
\hline T56-1 & Mercury in thermometer & 19 & 13 & $6(0.24)$ & $2.3(0.02)^{*}$ \\
\hline
\end{tabular}




\begin{tabular}{|c|c|c|c|c|c|}
\hline & & $(0.43)$ & $(0.71)$ & & \\
\hline T58-2 & $\begin{array}{l}\text { Incomplete combustion of } \\
\text { carbonaceous materials }\end{array}$ & $\begin{array}{l}99 \\
(2.26)\end{array}$ & $\begin{array}{l}39 \\
(2.14)\end{array}$ & $\begin{array}{l}60 \\
(2.35)\end{array}$ & $\begin{array}{l}0.5 \\
(0.6453)^{\mathrm{NS}}\end{array}$ \\
\hline $\begin{array}{l}\text { T59-4 } \\
\text { T59-6 }\end{array}$ & $\begin{array}{l}\text { Chlorine } \\
\text { Hydrogen sulfide gases }\end{array}$ & $\begin{array}{l}19 \\
(0.43)\end{array}$ & $7(0.38)$ & $\begin{array}{l}12 \\
(0.47)\end{array}$ & $\begin{array}{l}0.4 \\
(0.6549)^{\mathrm{NS}}\end{array}$ \\
\hline T60-0 & Organophosphate and Carbamates & $\begin{array}{l}885 \\
(20.24)\end{array}$ & $\begin{array}{l}255 \\
(14.01)\end{array}$ & $\begin{array}{l}630 \\
(24.69)\end{array}$ & $\begin{array}{l}8.7 \\
(0.0000)^{*}\end{array}$ \\
\hline T61-0 & Ciguatera & $\begin{array}{l}11 \\
(0.25)\end{array}$ & $5(0.27)$ & $6(0.24)$ & $\begin{array}{l}0.2 \\
(0.8455)^{\mathrm{NS}}\end{array}$ \\
\hline T62-2 & Castor beans and Datura seeds & $9(0.21)$ & $4(0.22)$ & $5(0.20)$ & $\begin{array}{l}0.1 \\
(0.8863)^{\mathrm{NS}}\end{array}$ \\
\hline T63-0 & Snake bites & $\begin{array}{l}54 \\
(1.24)\end{array}$ & $\begin{array}{l}31 \\
(1.70)\end{array}$ & $\begin{array}{l}23 \\
(0.90)\end{array}$ & $\begin{array}{l}2.4 \\
(0.0181)^{*}\end{array}$ \\
\hline
\end{tabular}

$@=$ Percentage in relation to $4372(100 \%)$ cases; \#=Percentage in relation to 1820 (100\%) cases; $\$=$ Percentage in relation to 2552 (100\%) cases; P=Probability; *=Significant difference at the 0.05 level; NS=No significant difference. 
Table (5): The percentage of distribution pattern of the clinical findings regarding overall cases and both genders.

\begin{tabular}{|c|c|c|c|c|}
\hline \multirow[b]{2}{*}{ Variables } & Overall & Males & Females & \multirow[b]{2}{*}{ Z-value $(\mathrm{P})$} \\
\hline & $\begin{array}{c}\text { Number } \\
(\%)^{@}\end{array}$ & $\begin{array}{c}\text { Number } \\
(\%)^{\#}\end{array}$ & $\begin{array}{l}\text { Number } \\
(\%)^{\$}\end{array}$ & \\
\hline \multicolumn{5}{|l|}{ PSS (Grades) } \\
\hline None (0) & $228(5.22)$ & $103(5.66)$ & $125(4.90)$ & $\begin{array}{l}1.1 \\
(0.2653)^{\mathrm{NS}}\end{array}$ \\
\hline Mild (1) & $\begin{array}{l}3372 \\
(77.13)\end{array}$ & $\begin{array}{l}1311 \\
(72.03)\end{array}$ & $\begin{array}{l}2061 \\
(80.76)\end{array}$ & $6.8(0.0001)^{*}$ \\
\hline Moderate (2) & $427(9.77)$ & $202(11.10)$ & $225(8.82)$ & $2.5(0.0123)^{*}$ \\
\hline Severe (3) & $308(7.04)$ & $177(9.73)$ & $131(5.13)$ & $5.9(0.0001)^{*}$ \\
\hline Fatal (4) & $37(0.85)$ & $27(1.48)$ & $10(0.39)$ & $3.9(0.0001)^{*}$ \\
\hline \multicolumn{5}{|l|}{ Ward admission } \\
\hline Emergency room & $\begin{array}{l}3074 \\
(70.31)\end{array}$ & $\begin{array}{l}1231 \\
(67.64)\end{array}$ & $\begin{array}{l}1843 \\
(72.22)\end{array}$ & $3.3(0.0011)^{*}$ \\
\hline Inpatient & $\begin{array}{l}1071 \\
(24.50)\end{array}$ & $439(24.12)$ & $632(24.76)$ & $\begin{array}{l}0.5 \\
(0.6276)^{\mathrm{NS}}\end{array}$ \\
\hline Intensive care unit & $227(5.19)$ & $150(8.24)$ & $77(3.02)$ & $7.7(0.0001)^{*}$ \\
\hline \multicolumn{5}{|l|}{ Hospital stay (Days) } \\
\hline$\leq 1$ day & $\begin{array}{l}3200 \\
(73.19)\end{array}$ & $\begin{array}{l}1310 \\
(71.98)\end{array}$ & $\begin{array}{l}1890 \\
(74.06)\end{array}$ & $\begin{array}{l}1.5 \\
(0.1259)^{\mathrm{NS}}\end{array}$ \\
\hline 2 days & $611(13.98)$ & $198(10.88)$ & $413(16.18)$ & $5(0.0001)^{*}$ \\
\hline 3 days & $299(6.84)$ & $131(7.20)$ & $168(6.58)$ & $\begin{array}{l}0.8 \\
(0.4233)^{\mathrm{NS}}\end{array}$ \\
\hline 4 days & $165(3.77)$ & $116(6.37)$ & $49(1.92)$ & $7.6(0.0001)^{*}$ \\
\hline$\geq 5$ days & $97(2.22)$ & $65(3.57)$ & $32(1.25)$ & $5.1(0.0001)^{*}$ \\
\hline \multicolumn{5}{|l|}{ Outcome } \\
\hline Complete recovery & $\begin{array}{l}4272 \\
(97.71)\end{array}$ & $\begin{array}{l}1752 \\
(96.26)\end{array}$ & $\begin{array}{l}2520 \\
(98.75)\end{array}$ & $5.4(0.0001)^{*}$ \\
\hline Nonfatal sequelae & $63(1.44)$ & $41(2.25)$ & $22(0.86)$ & $3.8(0.0001)^{*}$ \\
\hline \multicolumn{5}{|l|}{ Substances-induced death } \\
\hline $\begin{array}{l}\text { Organophosphate } \\
\text { Insecticides }\end{array}$ & $14(0.32)$ & $10(0.55)$ & $4(0.16)$ & $2.2(0.0249)^{*}$ \\
\hline Kerosene & $7(0.16)$ & $5(0.27)$ & $2(0.08)$ & $\begin{array}{l}1.6 \\
(0.1202)^{\mathrm{NS}}\end{array}$ \\
\hline Snake bite & $6(0.14)$ & $5(0.27)$ & $1(0.04)$ & $2(0.0417)^{*}$ \\
\hline Phenol & $5(0.11)$ & $2(0.11)$ & $3(0.12)$ & $\begin{array}{l}0.1 \\
(0.9237)^{\mathrm{NS}}\end{array}$ \\
\hline Opioids & $5(0.11)$ & $5(0.27)$ & $0(0.00)$ & $2.6(0.0086)^{*}$ \\
\hline
\end{tabular}

$@=$ Percentage in relation to $4372(100 \%)$ cases; \#=Percentage in relation to 1820 $(100 \%)$ cases; $\$=$ Percentage in relation to $2552(100 \%)$ cases; P=Probability; *=Significant difference at the 0.05 level; NS=No significant difference, PSS=Poisoning severity score; $\leq=$ Less than or equal to; $\geq=$ Greater than or equal to. 
Table (6): The percentage of distribution pattern of the most common therapeutic interventions regarding overall cases and both genders.

\begin{tabular}{|c|c|c|c|c|}
\hline \multirow[b]{2}{*}{ Variables } & Overall & Males & Females & \multirow[b]{2}{*}{$\begin{array}{c}\text { Z-value } \\
(\mathrm{P})\end{array}$} \\
\hline & $\begin{array}{c}\text { Number } \\
(\%)^{@}\end{array}$ & $\begin{array}{l}\text { Number } \\
(\%)^{\#}\end{array}$ & $\begin{array}{l}\text { Number } \\
(\%)^{\$}\end{array}$ & \\
\hline Decontamination & $\begin{array}{l}2944 \\
(67.34)\end{array}$ & $\begin{array}{l}1408 \\
(77.36)\end{array}$ & $\begin{array}{l}1536 \\
(60.19)\end{array}$ & $\begin{array}{l}11.9 \\
(0.0000)^{*}\end{array}$ \\
\hline External & $\begin{array}{l}113 \\
(2.58)\end{array}$ & $\begin{array}{l}47 \\
(2.58)\end{array}$ & $\begin{array}{l}66 \\
(2.59)\end{array}$ & $\begin{array}{l}0.02 \\
(0.9836)^{\mathrm{NS}}\end{array}$ \\
\hline Internal & $\begin{array}{l}2831 \\
(64.75)\end{array}$ & $\begin{array}{l}1361 \\
(74.78)\end{array}$ & $\begin{array}{l}1470 \\
(57.60)\end{array}$ & $\begin{array}{l}11.7 \\
(0.0000)^{*}\end{array}$ \\
\hline $\begin{array}{l}\text { All cases received activated } \\
\text { charcoal }\end{array}$ & $\begin{array}{l}1590 \\
(36.37)\end{array}$ & $\begin{array}{l}744 \\
(40.88)\end{array}$ & $\begin{array}{l}846 \\
(33.15)\end{array}$ & $\begin{array}{l}5.2 \\
(0.0001)^{*}\end{array}$ \\
\hline All cases received gastric lavage & $\begin{array}{l}989 \\
(22.62)\end{array}$ & $\begin{array}{l}557 \\
(30.60)\end{array}$ & $\begin{array}{l}432 \\
(16.93)\end{array}$ & $\begin{array}{l}10.6 \\
(0.0000)^{*}\end{array}$ \\
\hline All cases received syrup of Ipecac & $\begin{array}{l}618 \\
(14.14)\end{array}$ & $\begin{array}{l}212 \\
(11.65)\end{array}$ & $\begin{array}{l}406 \\
(15.91)\end{array}$ & $\begin{array}{l}4 \\
(0.0001)^{*}\end{array}$ \\
\hline Single method & $\begin{array}{l}2214 \\
(50.64)\end{array}$ & $\begin{array}{l}1075 \\
(59.07)\end{array}$ & $\begin{array}{l}1139 \\
(44.63)\end{array}$ & $\begin{array}{l}9.4 \\
(0.0000)^{*}\end{array}$ \\
\hline Activated charcoal & $\begin{array}{l}973 \\
(22.26)\end{array}$ & $\begin{array}{l}458 \\
(25.16)\end{array}$ & $\begin{array}{l}515 \\
(20.18) \\
\end{array}$ & $\begin{array}{l}3.9 \\
(0.0001)^{*}\end{array}$ \\
\hline Gastric lavage & $\begin{array}{l}703 \\
(16.08)\end{array}$ & $\begin{array}{l}392 \\
(21.54)\end{array}$ & $\begin{array}{l}311 \\
(12.19)\end{array}$ & $\begin{array}{l}8.3 \\
(0.0000)^{*}\end{array}$ \\
\hline Syrup of Ipecac & $\begin{array}{l}416 \\
(9.52)\end{array}$ & $\begin{array}{l}149 \\
(8.19)\end{array}$ & $\begin{array}{l}267 \\
(10.46)\end{array}$ & $\begin{array}{l}2.5 \\
(0.0117)^{*}\end{array}$ \\
\hline Demulcents & $\begin{array}{l}122 \\
(2.79)\end{array}$ & $\begin{array}{l}76 \\
(4.18)\end{array}$ & $\begin{array}{l}46 \\
(1.80)\end{array}$ & $\begin{array}{l}4.7 \\
(0.0001)^{*}\end{array}$ \\
\hline $\begin{array}{l}\text { Two methods (GIT evacuation + } \\
\text { Activated charcoal) }\end{array}$ & $\begin{array}{l}617 \\
(14.11)\end{array}$ & $\begin{array}{l}286 \\
(15.71)\end{array}$ & $\begin{array}{l}331 \\
(12.97)\end{array}$ & $\begin{array}{l}2.6 \\
(0.0103)^{*}\end{array}$ \\
\hline $\begin{array}{l}\text { Gastric lavage + Activated } \\
\text { charcoal }\end{array}$ & $\begin{array}{l}286 \\
(6.54)\end{array}$ & $\begin{array}{l}165 \\
(9.07)\end{array}$ & $\begin{array}{l}121 \\
(4.74)\end{array}$ & $\begin{array}{l}5.7 \\
(0.0001)^{*}\end{array}$ \\
\hline $\begin{array}{l}\text { Syrup of Ipecac + Activated } \\
\text { charcoal }\end{array}$ & $\begin{array}{l}202 \\
(4.62)\end{array}$ & $\begin{array}{l}63 \\
(3.46)\end{array}$ & $\begin{array}{l}139 \\
(5.45)\end{array}$ & $\begin{array}{l}3.1 \\
(0.002)^{*}\end{array}$ \\
\hline $\begin{array}{l}\text { Activated charcoal + Cathartic } \\
\text { Sorbitol }\end{array}$ & $\begin{array}{l}129 \\
(2.95)\end{array}$ & $\begin{array}{l}58 \\
(3.19) \\
\end{array}$ & $\begin{array}{l}71 \\
(2.78) \\
\end{array}$ & $\begin{array}{l}0.8 \\
(0.4297)^{\mathrm{NS}}\end{array}$ \\
\hline All administered specific antidotes & $\begin{array}{l}1104 \\
(25.25)\end{array}$ & $\begin{array}{l}689 \\
(37.86)\end{array}$ & $\begin{array}{l}415 \\
(16.26)\end{array}$ & $\begin{array}{l}16.2 \\
(0.0000)^{*}\end{array}$ \\
\hline Atropine & $\begin{array}{l}572 \\
(13.08)\end{array}$ & $\begin{array}{l}396 \\
(21.76)\end{array}$ & $\begin{array}{l}176 \\
(6.90)\end{array}$ & $\begin{array}{l}14.4 \\
(0.0000)^{*}\end{array}$ \\
\hline $\begin{array}{l}\text { Atropine + Obidoxime chloride } \\
\text { (Toxogonin) }\end{array}$ & $\begin{array}{l}171 \\
(3.91)\end{array}$ & $\begin{array}{l}101 \\
(5.55)\end{array}$ & $\begin{array}{l}70 \\
(2.74)\end{array}$ & $\begin{array}{l}4.7 \\
(0.0001)^{*}\end{array}$ \\
\hline Sodium bicarbonate & $\begin{array}{l}94 \\
(2.15)\end{array}$ & $\begin{array}{l}44 \\
(2.42)\end{array}$ & $\begin{array}{l}50 \\
(1.96)\end{array}$ & $\begin{array}{l}1 \\
(0.3014)^{\mathrm{NS}}\end{array}$ \\
\hline $\mathrm{N}$-acetylcysteine & $\begin{array}{l}81 \\
(1.85)\end{array}$ & $\begin{array}{l}35 \\
(1.92)\end{array}$ & $\begin{array}{l}46 \\
(1.80)\end{array}$ & $\begin{array}{l}0.3 \\
(0.7716)^{\mathrm{NS}}\end{array}$ \\
\hline
\end{tabular}




\begin{tabular}{|c|c|c|c|c|}
\hline Naloxone & $\begin{array}{l}52 \\
(1.19)\end{array}$ & $\begin{array}{l}40 \\
(2.20)\end{array}$ & $\begin{array}{l}12 \\
(0.47)\end{array}$ & $\begin{array}{l}5.2 \\
(0.0001)^{*}\end{array}$ \\
\hline Anticoagulant and Vitamin $\mathrm{K}$ & $\begin{array}{l}43 \\
(0.98)\end{array}$ & $\begin{array}{l}24 \\
(1.32)\end{array}$ & $\begin{array}{l}19 \\
(0.74)\end{array}$ & $\begin{array}{l}1.9 \\
(0.0552)^{\mathrm{NS}}\end{array}$ \\
\hline Glucose & $\begin{array}{l}35 \\
(0.80)\end{array}$ & $\begin{array}{l}15 \\
(0.82)\end{array}$ & $\begin{array}{l}20 \\
(0.78)\end{array}$ & $\begin{array}{l}0.1 \\
(0.8834)^{\mathrm{NS}}\end{array}$ \\
\hline Polyvalent snake antivenom & $\begin{array}{l}19 \\
(0.43)\end{array}$ & $\begin{array}{l}13 \\
(0.71)\end{array}$ & $6(0.24)$ & $2.3(0.02)^{*}$ \\
\hline Flumazenil & $\begin{array}{l}16 \\
(0.37)\end{array}$ & $7(0.38)$ & $9(0.35)$ & $\begin{array}{l}0.2 \\
(0.8708)^{\mathrm{NS}}\end{array}$ \\
\hline Scorpion antivenom & $9(0.21)$ & $6(0.33)$ & $3(0.12)$ & $\begin{array}{l}1.5 \\
(0.1325)^{\mathrm{NS}}\end{array}$ \\
\hline Other & $\begin{array}{l}12 \\
(0.27)\end{array}$ & $8(0.44)$ & $4(0.16)$ & $\begin{array}{l}1.7 \\
(0.0822)^{\mathrm{NS}}\end{array}$ \\
\hline Symptomatic treatment & $\begin{array}{l}788 \\
(18.02)\end{array}$ & $\begin{array}{l}530 \\
(29.12) \\
\end{array}$ & $\begin{array}{l}258 \\
(10.11)\end{array}$ & $\begin{array}{l}16.1 \\
(0.0000)^{*}\end{array}$ \\
\hline Emergent and Supportive measures & $\begin{array}{l}399 \\
(9.13)\end{array}$ & $\begin{array}{l}289 \\
(15.88)\end{array}$ & $\begin{array}{l}110 \\
(4.31)\end{array}$ & $\begin{array}{l}13.1 \\
(0.0000)^{*}\end{array}$ \\
\hline
\end{tabular}

$@=$ Percentage in relation to $4372(100 \%)$ cases; \#=Percentage in relation to 1820 $(\mathbf{1 0 0 \%})$ cases; $\$=$ Percentage in relation to 2552 (100\%) cases; $P=P r o b a b i l i t y ;$ *=Significant difference at the 0.05 level; NS=No significant difference; GIT $=$ Gastrointestinal tract.

\section{DISCUSSION}

Acute poisoning is a growing worldwide problematic phenomenon that has enormous negative influences on the individuals and communities. Its incidence and characteristics varies significantly between countries and different regions within the same country (Obeidat et al., 2010; Sawalha et al., 2010; Jaiprakash et al., 2011), including Egypt (El Masry and Tawfik, 2013; Hassan and Siam, 2014). To understand the magnitude of this problem, different epidemiological information about poisoning profiles is required. Hence, the present study was conducted to analyze the pattern of acute poisoning in Benha city and its surrounding areas in order to initiate preventive measures.

Similar to the present gender result, previous studies conducted in other part of Egypt (El Masry and Tawfik, 2013) and different countries (Aydin et al., 2014; Kara et al., 2014; Koylu et al., 2014) displayed more prevalence of intoxication among females. However, other studies illustrated higher figures among males (Jaiprakash et al., 2011; Khudair et al., 2013) or almost equal ratio between genders (Moghadamnia and Abdollahi, 2002).

Analogous to the current findings of age distribution, preceding results showed predominance of poisoning among patients younger than 20-yearas well as a bimodal pattern of distribution with the first peak in children <6-year and the second peak in adults aged between 20-29-year (Obeidat et al., 2010; Mowry et al., 2015), in addition, males <20-year (Obeidat et al., 2010; Mowry et al., 2015), boys <6-year (El Masry and Tawfik, 2013; Ozdogan et 
al., 2008), and females in almost all age groups (Kara et al., 2014; Koylu et al., 2014) were predominantly affected.

On the Contrary, other studies showed that most of the patients were in the age group $>18$-year, especially those in the age category between 18-29-year (Afshari et al., 2004; Ahmadi et al., 2010) or between 15-24-year (Tüfekçi et al., 2004), with predominance of males (Sawalha et al., 2010) or females (Akbaba et al., 2007) in all age groups. Also, distribution of gender was nearly equal between boys and girls (Mert and Bilgin, 2006).

Frequent poisoning casualty in children can be explained by tremendous sense of curiosity, increased activity, oral identification of substances, simulating adults, and recognition inability of harmful agents (Presgrave et al., 2008; El Masry and Tawfik, 2013) as well as easy accessibility to toxic products due to improper storage and packaging of different products and inadequate supervision of the children by the parents or caregivers (Obeidat et al., 2010). The higher rate of poisoning during the third decade of life might be due to living under life settlement stressful conditions with subsequent development of substantial amount of physical, social, and mental disorders (Karki and Risal, 2012).

In agreement with the existing results of the socio-epidemiological patterns, several authors found that the majority of patients were unmarried (Hatzitolios et al., 2001; Güloğlu and Kara, 2005; Rajapakse et al., 2014), rural residents (Karki and Risal, 2012), of both low economic and educational levels (not enrolled in university), household workers (Sabzghabaee et al., 2013), actually or potentially unemployed, and previously healthy with infrequent existence of mental and physical disorders (Azizpour et al., 2016) and other diseases (Akbaba et al., 2007).

However, the aforementioned results are not in agreement with those of several other authors, where most of the patients were married (Baydin et al., 2005; Jaiprakash et al., 2011) or nearly equal in marital status, urban residents (Sabzghabaee et al., 2013), of upper middle income level (Jaiprakash et al., 2011), with primary education level (Rajapakse et al., 2014), employed (Hatzitolios et al., 2001), and suffering from psychiatric disorders (Baydin et al., 2005).

Parallel with the present results of gender distribution, other investigators reported that poisoned males were chiefly married (Jaiprakash et al., 2011) and of low income level (Thanh et al., 2005), whereas females were largely unmarried (Kara et al., 2014), rural residents (Thanh et al., 2005), enrolled in all levels of education, unemployed (Rajapakse et al., 2014), and suffering from health disorders (Thanh et al., 2005).

Contrariwise to this, other studies showed that males were mainly unmarried (Jaiprakash et al., 2011), rural residents (Kanchan and Menezes, 2008), and employed (Rajapakse et al., 2014) or unemployed (Hatzitolios et al., 2001).

It has been speculated that single individuals are more prone to psychological trauma than married persons and inability to cope with pressures of marriage problems is upsetting stressful events that may increase rates of mood disorders (Mert and Bilgin, 2006). Moreover, persons residing in rural regions commonly 
suffer from chronic socio-economic problems and environmental difficulties (Jesslin et al., 2010). Furthermore, inability to deal with tensions of economic hardship due to insufficient financial support or idleness (Jaiprakash et al., 2011) and failure to achieve remarkable targets of educational attainment or good school reports (Mert and Bilgin, 2006) might be leading reasons to commit poisoning actions.

Compatible with the current findings of the overall toxicological profiles, other published studies displayed that the vast majority of cases were exposed to poisons at homes (Tüfekçiet al., 2004; Hung et al., 2008), and arrived to the hospital within 3 hours after poisoning events (Avsarogullari et al., 2012; Kara et al., 2014), essentially during afternoon shift (Hatzitolios et al., 2001). Also, poisoning episodes predominantly peaked in the summer season and declined in the winter season (Akbaba et al., 2007; Azizpour et al., 2016), occurred via oral route followed by inhalation, and unintentional manner (Moazzam et al., 2009; Khudair et al., 2013) with nil homicidal cases (Moghadamnia and Abdollahi, 2002), and caused by single product (Ahmadi et al., 2010; Sorodoc et al., 2011).

On the contrary, different results were reported in several other studies, where Poison Control or Information Centers received calls mostly from general public and private health services (Bentur et al., 2004). Furthermore, most of the patients were arrived to the hospital during the first two hours (Akkas et al., 2004) or after 7 hours (Satar and Seydaoglu, 2005) of poisoning events and hospitalized during evening shift (Azizpour et al.,
2016). Also, the incidences of poisoning were mostly increased in spring and decreased in autumn months (Islambulchilar et al., 2009), occurred via skin puncture (animal bites and stings and intramuscular or intravenous) (Lall et al., 2003), and intentional (Güloğlu and Kara, 2005; Jaiprakash et al., 2011) with some criminal manner of poisoning (Afshari et al., 2004; El Masry and Tawfik, 2013).

Several authors suggested that excessive availability of poisonous substances with easy accessibility inside domestic environment (Mert and Bilgin, 2006), visiting other health care institutions or long referral distance before hospital admission, lack of parental or caregiver supervision of children, high incidence of family quarrels, and domestic troubles during afternoon and evening hours (Karki and Risal, 2012), mood fluctuations, holiday problems, and hormonal changes during summer and spring months (Mert and Bilgin, 2006), and the vast majority of poisonous substances found in solid and liquid forms (Moazzam et al., 2009) might be contributory factors for the previous findings. Also, traditions and increased spinsterhood, social and family problems, and emotional abuse may be the main causes of suicidal attempts among females (Aboul-Hagag et al., 2013), while males tend to use more violent methods to commit suicide than self-poisoning (Abdel Moneim et al., 2012).

Symmetric with the present findings of gender distribution, many other studies exhibited that males were more significantly exposed to poisoning at outdoor (Rajapakse et al., 2014), during evening and night hours (Kanchan and Menezes, 2008) and 
spring season (Räsänen et al., 2002), via skin puncture (Jesslin et al., 2010), and via accidental manner (Bentur et al., 2004; Khudair et al., 2013), while females were more predominantly exposed to intoxication during morning and afternoon hours (Kanchan and Menezes, 2008), summer months (Coakley et al., 1994), and via intentional or suicidal manner (Kara et al., 2014; Koylu et al., 2014; Rajapakse et al., 2014).

However, the prior findings were inconsistent with those of other publications, where females mostly showed a lag time for 3 hours before hospital arrival (Kara et al., 2014), nonexposure to poisons during night hours (Kanchan and Menezes, 2008), and autumn peaks (Räsänen et al., 2002). Also, intentional and unintentional poisoning were commonly reported among males (van der Hoek and Konradsen, 2005) and females (Güloğlu and Kara, 2005), respectively, or unintentional manner was nearly equally distributed between genders (Akbaba et al., 2007). Additionally, exposure to single as well as multiple agents occurred more frequently among females (Aydin et al., 2014).

The distribution pattern of medicinal and non-medicinal groups and their causative agents differs significantly and mixed results were reported between studies. Concordant with the existing results of the implicated poisonous groups, other researches authenticated that compounds categorized in nonmedicinal group were chiefly responsible for the majority of poisoning, especially single causative agent (Lall et al., 2003; Srivastava et al., 2005; Hung et al., 2008). Also, exposure to single agent predominated over multiple agents (Satar and Seydaoglu, 2005; Aydin et al., 2014).

However, paradoxical results were recorded by several authors who found that preparations listed in the medicinal group were the most common cause of poisoning (Tüfekçi et al., 2004; Baydin et al., 2005; Güloğlu and Kara, 2005), while in Egypt (El Masry and Tawfik, 2013) and United states (Mowry et al., 2015) the ratio between both groups was almost equal.

In accordance with the present results of gender distribution, former studies revealed a slight predominance of poisoning with substances listed in medicinal group among males (Hassanian-Moghaddam et al., 2014), while females were more frequently exposed to substances listed in nonmedicinal group (Aydin et al., 2014).

Opposite to this, many reports showed that males were mostly exposed to substances listed in non-medicinal group, whereas females were more frequently exposed to substances listed in medicinal group (Hanssens et al., 2001; Mert and Bilgin, 2006; Rajasuriar et al., 2007; Khudair et al., 2013). These discrepancies could be linked to cultural background and variability in manner of exposure between genders as well as different regions in Egypt and other countries.

The current distribution patterns of non-medicinal-related poisoning were comparable with those of most other studies, which manifested that the top five non-medicinal groups mostly implicated in poisoning among this group, in order of frequency, were pesticides, corrosive substances (Satar and Seydaoglu, 2005; Srivastava et al., 2005; Islambulchilar et al., 2009; Aydin et al., 2014), organic solvents, 
carbon monoxide (Khudair et al., 2013), and alcohol (Avsarogullari et al., 2012). Among the previous groups, the major involved causative agents within each subgroup were, respectively, organophosphate and carbamate (Afshari et al., 2004; El Masry and Tawfik, 2013), alkalis (Tüfekçi et al., 2004), kerosene (Lall et al., 2003; Afshari et al., 2004), carbon monoxide due to coal or wood stoves dysfunction (Tüfekçi et al., 2004), and ethanol (Akkose et al., 2005).

On the contrary, the ranking order of the present involved non-medicinal groups was completely inconsistent with other studies conducted in Iran (Hassanian-Moghaddam et al., 2014) and Malaysia (Rajasuriar et al., 2007) (except for pesticide group). Also, exposures to gaseous agents (Tüfekçi et al., 2004), corrosives (Mert and Bilgin, 2006), noxious food (Akkose et al., 2005), alcohols (Khudair et al., 2013), or bites/stings (Lall et al., 2003) were recorded as the primary involved nonmedicinal groups in poisoning incidents. Furthermore, poisoning with agents such as aluminium phosphide (Srivastava et al., 2005), rodenticides (Aydin et al., 2014), caustic acids (Afshari et al., 2004), methanol (Shadnia et al., 2007), and scorpion stings (Lall et al., 2003) were more frequently reported in other studies, which disagree with this findings.

The present results of nonmedicinal-related poisoning among genders were also analogous with those of other studies, which elucidated predominance of poisoning with alcohol and animal envenomation (Obeidatet al., 2010; Hassanian-Moghaddam et al., 2014) among males, while poisoning with pesticides, corrosives, and carbon monoxide were prevalent in females
(Tüfekçi et al., 2004; Aydin et al., 2014; Kara et al., 2014).

On the contrary, poisoning with pesticides, corrosives (Mert and Bilgin, 2006), and carbon monoxide (Hassanian-Moghaddam et al., 2014) were commonly noticed among males, whereas poisoning with corrosives was almost equal between genders (Akkose et al., 2005). These diversities may be due to differences in choice of poisoning agents according to their availabilities for both genders as well as between various countries.

The present distribution patterns of medicinal-related poisoning were consistent with those of most other studies, which exhibited that the top five medicinal groups most commonly implicated in poisoning among this group, in order of frequency, were reported in classes T42 (antiepileptic, sedative-hypnotic and antiparkinsonism), T39 (non-opioid analgesics, antipyretics, and antirheumatics) (Moghadamnia and Abdollahi, 2002; Srivastava et al., 2005; Hassanian-Moghaddam et al., 2014), T36 (systemic antibiotics) (Akkas et al., 2004), T40 (narcotics and psychodysleptics)

(HassanianMoghaddam et al., 2014), and T48 (agents primarily acting on muscles and the respiratory system) (Hanssens et al., 2001). Among the previous groups, the major causative agents within each subgroup were, respectively, sedativehypnotics and benzodiazepine derivatives (Afshari et al., 2004; Ahmadi et al., 2010; Sorodoc et al., 2011), acetaminophen and NSAIDs (Moghadamnia and Abdollahi, 2002; Lall et al., 2003; El Masry and Tawfik, 2013), penicillins (Afshari et al., 2004), cannabis (El Masry and Tawfik, 2013; Hassanian- 
Moghaddam et al., 2014) and tramadol and theophylline (El Masry and Tawfik, 2013), which are similar to the present results.

However, contradictory results were noticed in numerous other studies, where non-opioid analgesics (Hanssens et al., 2001; Hatzitolios et al., 2001), opioid analgesics (Afshari et al., 2004), antidepressants (Tüfekçi et al., 2004), or psychoactive drugs (Güloğlu and Kara, 2005) constituted the first classes among drug-related poisoning. Also, poisoning with opioids (Hatzitolios et al., 2001) and benzodiazepines (Khudair et al., 2013), respectively, were more and less frequently recorded in many studies than this work.

The existing findings of medicinalrelated poisoning among genders were also in accordance with those of other previous studies, which demonstrated predominance of poisoning with antibiotics (Hanssens et al., 2001) and narcotics and psychodysleptics (Hassanian-Moghaddam et al., 2014; Aydin et al., 2014) drugs among males, while poisoning with sedativehypnotics,non-opioid analgesics

(Sorodoc et al., 2011), and autonomic nervous system drugs (HassanianMoghaddam et al., 2014) were commonly reported among females.

Contradictory to the current findings, poisoning with non-opioid analgesics, antipyretics and antirheumatics were frequently noticed among males, while poisoning with antibiotic, antidepressant, and gastrointestinal drugs were mostly seen among females (HassanianMoghaddam et al., 2014).

The current results of poisoning severity were markedly in alignment with those of other investigators who found that the vast majority of cases commonly experienced mild clinical manifestations (Hung et al., 2008; El Masry and Tawfik, 2013) and their clinical status were generally good on arrival to the hospital with most of the patients were mainly admitted in the emergency department (Baydin et al., 2005; Avsarogullari et al., 2012) and discharged from hospital within 24-hour of follow-up period without any morbidity (Ozdogan et al., 2008; Sabzghabaee et al., 2013). Furthermore, the mortality rate was considerably low (less than 1\%) (Akkas et al., 2004; El Masry and Tawfik, 2013) and pesticide compounds were the predominant cause of death (Hatzitolios et al., 2001; El Masry and Tawfik, 2013).

In contrast to the previous findings, it was found that the main bulk of patients were asymptomatic (Bentur et al., 2004), hospitalized and monitored for more than 24-hour (Satar and Seydaoglu, 2005). Also, many reports showed that the fatality rate was nil (Lall et al., 2003) or high (more than 1\%) (Islambulchilar et al., 2009; Jaiprakash et al., 2011) and opioids (Shadnia et al., 2007) or drugs (Afshari et al., 2004) were the commonest cause of death.

The existing results of clinical status among genders were also parallel with those of other studies, which illustrated more prevalence of severe toxidromes (Bentur et al., 2004), intensive care unit admission (Goksu et al., 2002), prolonged hospital stay (Satar and Seydaoglu, 2005), and fatality rate (Afshari et al., 2004; Mert and Bilgin, 2006) among male patients, whereas female patients mainly admitted to emergency department (Goksu et al., 2002). However, many authors had reported higher mortality rate among 
females rather than males (Ahmadi et al., 2010).

In general, poisoned patients are essentially treated by utilization of decontamination methods, specific antidotes, symptomatic treatment and emergent and supportive measures either alone or in combination. Internal decontamination procedures via utilizations of oral activated charcoal, gastric lavage, syrup of ipecac, and cathartic effectively remove stomach contents and decrease the systemic absorption of poisonous agents, especially when applied within 1-4 hours post ingestion (Hatzitolios et al., 2001; Mert and Bilgin, 2006; Khudair et al., 2013).

Similar to the present study, previous reports showed that initial treatment was given to all symptomatic patients and at least one method of therapeutic intervention was used with utilization of internal decontamination methods predominated over administration of specific antidotes and other therapies (Hatzitolios et al., 2001; Akkas et al., 2004; Baydin et al., 2005; El Masry and Tawfik, 2013).

However, utilization of internal decontamination methods varies between studies. In the present study, activated charcoal (a highly effective and less invasive decontamination procedure) was the most frequently applied method, which is almost similar to that of other study conducted in Vietnam (Hung et al., 2008). On the contrary, the current distribution patterns of therapeutic interventions were incomparable with those of other many studies, which exhibited that the most commonly applied methods were concomitant use of activated charcoal and cathartics (Hatzitolios et al., 2001), gastric lavage and activated charcoal
(Akkas et al., 2004; Baydin et al., 2005), and gastric lavage, activated charcoal, and cathartics (Tüfekçi et al., 2004), performance of gastric lavage alone (Satar and Seydaoglu, 2005), and induction of emesis by ipecac syrup (Moghadamnia and Abdollahi, 2002; El Masry and Tawfik, 2013), while in other studies performance of gastric lavage, administration of activated charcoal (Sawalha et al., 2010; El Masry and Tawfik, 2013), and the use of ipecac syrup (Hatzitolios et al., 2001) were less frequently applied than this study. Also, several studies revealed that most of the cases did not receive any decontamination procedures and only managed by close medical observation in the emergency room (Satar and Seydaoglu, 2005; Sawalha et al., 2010; Khudair et al., 2013).

The present study has some limitations. This was a hospital-based retrospective study done at BPCU. Therefore, the study will not reflect the overall pattern of poisoning in the country. Also, cases deceased before reaching the hospital, seeking primary and secondary healthcare centers, and transferred to other poison control centers were not included. Moreover, lack of information about stressful events, incomplete data in some medical records, inability to identify some causative agents, and inability to follow up some cases after hospital discharge were noticed. Suicide is a legal offence in Egypt and some patients or attendants might deny suicidal intent to avoid legal repercussions. Thus, it is quite possible that some patients who were recorded as unintentional were actually attempted suicides.

In conclusion, the current study provides important information about the pattern of poisoning in Benha city 
and its surrounding areas. The majority of cases were females, under 18-year, unmarried, rural residents, of low economic and educational status, unemployed, and previously healthy. Also, pre-school children and young adults were the most vulnerable groups. Poisoning at homes, a prehospitalization period for 3-hour, and hospital admission during afternoon shift and summer season as well as exposures via oral route, unintentional manner, and single agent were mostly reported. A predominance of poisoning with substances listed in non-medicinal group was found. Among them, pesticides (particularly organophosphate and carbamates), followed by corrosives (particularly alkalis), and organic solvents (particularly kerosene) were the most common implicated causative agents. While, among the medicinalrelated poisoning, sedative-hypnotics (especially benzodiazepines), followed by non-opioid analgesics (especially acetaminophen and NSAIDs), and narcotics and hallucinogens (especially tramadol and cannabis) were the most common involved causative agents. The most frequently recorded clinical findings were mild toxidromes, emergency room admission, hospitalization for $\leq 1$-day, and complete recovery. Organophosphates were the chief cause of death. The vast majority of cases received different internal decontamination procedures followed by administration of specific antidotes, symptomatic treatment, and finally emergent and supportive measures. Comparison between genders revealed statically significant differences regarding some of the studied variables.

Concerning the present study, some suggestions are recommended. Restricted attention and supervision are needed for children. Patients with suicidal poisoning must undergo psychiatric consultation. Afternoon hours are very busy periods; hence, relief hours for medical staffs should be better during early evening hours. Strict regulation and control regarding the sale of pesticides and central nervous system drugs are crucial. Continuous health education and adequate information concerning hazardous effects of pesticides should be conducted. Potentially toxic drugs must not be sold without the prescription. To reduce morbidity and mortality associated poisoning, considerations must be given to establishing treatment guidelines, training healthcare providers, and ensuring sufficient stocks of the antidotes, especially atropine and toxogonin. The underlying causes of attempt suicide require further investigations.

\section{REFERENCES}

Abdel Moneim, W.M., Yassa, H.A., and George, S.M. (2012): Suicide trends in Upper Egypt. J Forensic Sci, 57:1247-1251.

Aboul-Hagag, K.E.S., Ahmed, F.A., Mansour, A.E. (2013): Forensic analysis of suicide mortality in Sohag governorate (Upper Egypt) in the period 2005-2009. Egyptian Journal of Forensic Sciences, 3:5360.

Afshari, R., Majdzadeh, R., and Balali-Mood, M. (2004): Pattern of acute poisonings in Mashhad, Iran 1993-2000. J Toxicol Clin Toxicol, 42:965-975.

Ahmadi, A., Pakravan, N., and Ghazizadeh, Z. (2010): Pattern of acute food, drug and chemical poisoning in Sari City, Northern Iran. Hum Exp Toxicol, 29:731-738. 
Akbaba, M., Nazlican, E., Demirhindi, H., Sütoluk, Z., and Gökel, Y. (2007): Etiological and demographical characteristics of acute adult poisoning in Adana, Turkey. Hum Exp Toxicol, 26:401406.

Akkas, M., Coskun, F., Ulu, N., and Sivri, B. (2004): An epidemiological evaluation of 1098 acute poisoning cases from Turkey. Vet Hum Toxicol, 46:213-215.

Akkose, S., Bulut, M., Armagan, E., Cebicci, H., and Fedakar, R. (2005): Acute poisoning in adults in the years 1996-2001 treated in the Uludag University Hospital, Marmara Region, Turkey. Clin Toxicol (Phila), 43:105-109.

Avsarogullari, L., Senol, V., Akdur, O., Akin, A., Durukan, P., and Ozkan, S. (2012): Characteristics of acute adult poisonings in a university hospital emergency department in central Turkey: a three-year analysis. J Pak Med Assoc, 62:129-133.

Aydin, I., Karadas, S., Gonullu, H., and Dulger, A.C. (2014): Epidemiological analysis of poisoning cases in Van, Turkey. $\mathrm{J}$ Pak Med Assoc, 64:560-562.

Azizpour, Y., Asadollahi, K., Sayehmiri, K., Kaikhavani, S., and Abangah, G. (2016): Epidemiological survey of intentional poisoning suicide during 1993-2013 in Ilam Province, Iran. BMC Public Health, 16:902.

Baydin, A., Yardan, T., Aygun, D., Doganay, Z., Nargis, C., Incealtin, O. (2005): Retrospective evaluation of emergency service patients with poisoning: a 3-year study. Adv Ther, 22:650-658.
Bentur, Y., Raikhlin-Eisenkraft, B., and Lavee, M. (2004): Toxicological features of deliberate self-poisonings. Hum Exp Toxicol, 23:331-337.

Coakley, F., Hayes, C., Fennell, J., and Johnson, Z. (1994): A study of deliberate self-poisoning in a Dublin hospital 1986-1990. Ir J Psychol Med, 11:70-72.

El Masry, M.K. and Tawfik, H.M. (2013): 2011 Annual report of the poison control centre of Ain Shams University Hospital, Cairo, Egypt. Ain Shams J Forensic Med Clin Toxicol, 20:10-17.

Goksu, S., Yildirim, C., Kocoglu, H., Tutak, A., and Oner, U. (2002): Characteristics of acute adult poisoning in Gaziantep, Turkey. J Toxicol Clin Toxicol, 40:833-837.

Güloğlu, C. and Kara, I.H. (2005): Acute poisoning cases admitted to a university hospital emergency department in Diyarbakir, Turkey. Hum Exp Toxicol, 24:49-54.

Hanssens, Y., Deleu, D., and Taqi, A. (2001): Etiologic and demographic characteristics of poisoning: a prospective hospital-based study in Oman. J Toxicol Clin Toxicol, 39:371-380.

Hassan, B.A. and Siam, M.G. (2014): Patterns of Acute Poisoning in Childhood in Zagazig, Egypt: An Epidemiological Study. Int Sch Res Notices, 2014:245279.

Hassanian-Moghaddam, H., Zamani, N., Rahimi, M., Shadnia, S., Pajoumand, A., and Sarjami, S. (2014): Acute adult and adolescent poisoning in Tehran, Iran; the epidemiologic trend between 2006 and 2011. Arch Iran Med, 17:534538 . 
Hatzitolios, A.I., Sion, M.L., Eleftheriadis, N.P., Toulis, E., Efstratiadis, G., Vartzopoulos, D., and Ziakas, A.G. (2001): Parasuicidal poisoning treated in a Greek medical ward: epidemiology and clinical experience. Hum Exp Toxicol, 20:611-617.

Hung, H.T. Du Nguyen, T., and Höjer J. (2008): The first poison control center in Vietnam: experiences of its initial years. Southeast Asian J Trop Med Public Health, 39:310-318.

Islambulchilar, M., Islambulchilar, Z., and Kargar-Maher, M.H. (2009): Acute adult poisoning cases admitted to a university hospital in Tabriz, Iran. Hum Exp Toxicol, 28:185-190.

Jaiprakash, H., Sarala, N., Venkatarathnamma, P.N., and Kumar, T.N. (2011): Analysis of different types of poisoning in a tertiary care hospital in rural South India. Food Chem Toxicol, 49:248250.

Jesslin, J., Adepu, R., and Churi, S. (2010): Assessment of prevalence and mortality incidences due to poisoning in a South Indian tertiary care teaching hospital. Indian J Pharm Sci, 72:587-591.

Kanchan, T. and Menezes, R.G. (2008): Suicidal poisoning in Southern India: gender differences. J Forensic Leg Med, 15:7-14.

Kara, H., Bayir, A., Degirmenci, S., Kayis, S.A., Akinci, M., Ak, A., Agacayak, A., and Azap, M. (2014): Causes of poisoning in patients evaluated in a hospital emergency department in Konya, Turkey. J Pak Med Assoc, 64:10421048.

Karki, R.K. and Risal, A. (2012): Study of poisoning cases in a tertiary care hospital. Kathmandu Univ Med J, 10:70-73.

Khudair, I.F., Jassim, Z., Hanssens, Y., and Alsaad, W.A. (2013): Characteristics and determinants of adult patients with acute poisoning attending the accident and emergency department of a teaching hospital in Qatar. Hum Exp Toxicol, 32:921-929.

Koylu, R., Dundar, Z.D., Koylu, O., Akinci, E., Akilli, N.B., Gonen, M.O., and Cander, B. (2014): The experiences in a toxicology unit: a review of 623 cases. J Clin Med Res, 6:59-65.

Lall, S.B., Al-Wahaibi, S.S., AlRiyami, M.M., and Al-Kharusi, K. (2003): Profile of acute poisoning cases presenting to health centres and hospitals in Oman. East Mediterr Health J, 9:944-954.

Mathers, C., Boerma, T., and Fat, D.M., (2008): Deaths by cause, sex and income group in WHO regions, estimates for 2004. In: The global burden of disease 2004 Update World Health Organization. Geneva 27, Switzerland: WHO Press, 2008, pp.74-83.

Mert, E. and Bilgin, N.G. (2006): Demographical, aetiological and clinical characteristics of poisonings in Mersin, Turkey. Hum Exp Toxicol, 25:217-223.

Moazzam, M., Al-Saigul, A.M., Naguib, M., and Al Alfi, M.A. (2009): Pattern of acute poisoning in Al-Qassim region: a surveillance report from Saudi Arabia, 19992003. East Mediterr Health J, 15:1005-1010.

Moghadamnia, A.A. and Abdollahi, M. (2002): An epidemiological study of poisoning in northern 
Islamic Republic of Iran. East Mediterr Health J, 8:88-94.

Mowry, J.B., Spyker, D.A., Brooks, D.E., McMillan, N., and Schauben, J.L. (2015): 2014 Annual Report of the American Association of Poison Control Centers' National Poison Data System (NPDS): 32nd Annual Report. Clin Toxicol (Phila), 53:962-1147.

Obeidat, N.M., Abutayeh, R.F., and Hadidi, K.A. (2010): Poisoning in Jordan: Analysis of Three Year Data from Jordan National Drug and Poison Information Center. J Med J, 44:298-304.

Ozdogan, H., Davutoglu, M., Bosnak, M., Tutanc, M., and Haspolat, K. (2008): Pediatric poisonings in southeast of Turkey: epidemiological and clinical aspects. Hum Exp Toxicol, 27:4548.

Persson, H.E., Sjöberg, G.K., Haines, J.A., and Pronczuk de Garbino, J. (1998): Poisoning severity score. Grading of acute poisoning. J Toxicol Clin Toxicol, 36:205-213.

PresgraveRde, F., Camacho, L.A., and Villas Boas, M.H. (2008): A profile of unintentional poisoning caused by household cleaning products, disinfectants and pesticides. Cad Saude Publica, 24:2901-2908.

Rajapakse, T., Griffiths, K.M., Christensen, H., and Cotton, S. (2014): A comparison of non-fatal self-poisoning among males and females, in Sri Lanka. BMC Psychiatry, 14:221.

Rajasuriar, R., Awang, R., Hashim, S.B., and Rahmat, H.R. (2007): Profile of poisoning admissions in
Malaysia. Hum Exp Toxicol, 26:7381.

Räsänen, P., Hakko, H., Jokelainen, J., and Tiihonen, J. (2002): Seasonal variation in specific methods of suicide: a national register study of 20,234 Finnish people. J Affect Disord, 71:51-59.

Sabzghabaee, A.M., Soleimani, M., Farajzadegan, Z., Hosseinpoor, S., Mirhosseini, S.M., and EizadiMood, N. (2013): Social risk factors and outcome analysis of poisoning in an Iranian referral medical center: A toxico-epidemiological approach. J Res Pharm Pract, 2:151-155.

Satar, S. and Seydaoglu, G. (2005): Analysis of acute adult poisoning in a 6-year period and factors affecting the hospital stay. Adv Ther, 22:137147.

Sawalha, A.F., Sweileh, W.M., Tufaha, M.T., and Al-Jabi, D.Y. (2010): Analysis of the pattern of acute poisoning in patients admitted to a governmental hospital in Palestine. Basic Clin Pharmacol Toxicol, 107:914-918.

Shadnia, S., Esmaily, H., Sasanian, G., Pajoumand, A., HassanianMoghaddam, H., and Abdollahi, M. (2007): Pattern of acute poisoning in Tehran-Iran in 2003. Hum Exp Toxicol, 26:753-756.

Sorodoc, V., Jaba, I.M., Lionte, C., Mungiu, O.C., and Sorodoc, L. (2011): Epidemiology of acute drug poisoning in a tertiary center from Iasi County, Romania. Hum Exp Toxicol, 30:1896-1903.

Srivastava, A., Peshin, SS., Kaleekal, T., and Gupta, S.K. (2005): An epidemiological study of poisoning cases reported to the National Poison Information Center, all India institute of medical sciences, New 
Delhi. Hum Exp Toxicol, 24:279285.

Taha, M.M., Abdel Aal, A.G.E., Ali, A.A.E., Mohamed, M.A., and Zaki, M.K. (2011): Suicide mortality in Cairo city, Egypt: A retrospective study. Egyptian Journal of Forensic Sciences, 1:3034.

Thanh, H.T., Jiang, G.X., Van, T.N., Minh, D.P., Rosling, H., and Wasserman, D. (2005): Attempted suicide in Hanoi, Vietnam. Soc
Psychiatry Psychiatr Epidemiol, 40:64-71.

Tüfekçi, I.B., Curgunlu, A., and Sirin, F. (2004): Characteristics of acute adult poisoning cases admitted to a university hospital in Istanbul. Hum Exp Toxicol, 23:347-351.

van der Hoek, W. and Konradsen, F. (2005): Risk factors for acute pesticide poisoning in Sri Lanka. Trop Med Int Health, 10:589-596. 


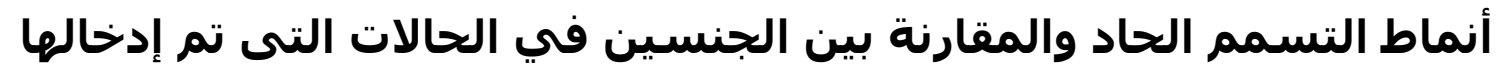

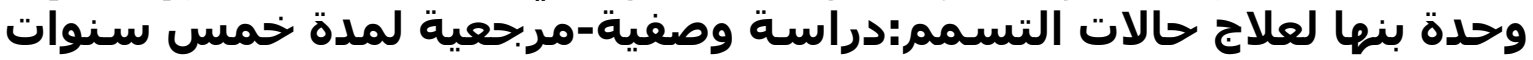 $(T \cdot 10-T \cdot 11)$
}

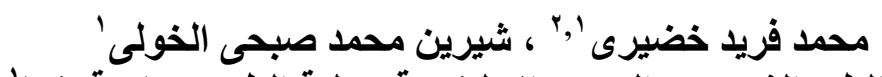

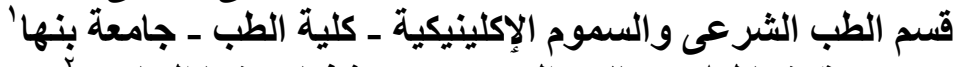

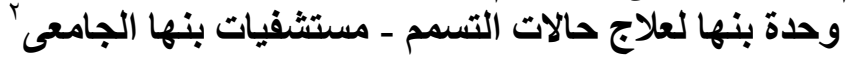

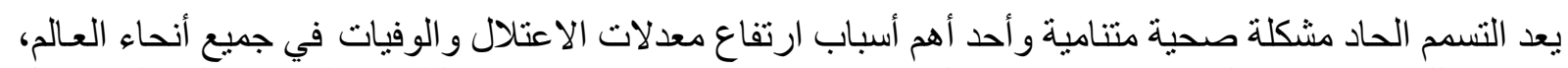

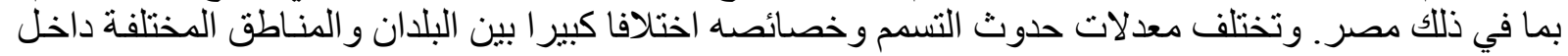

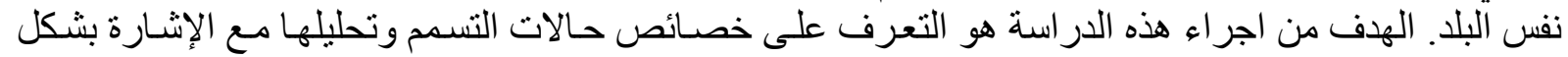

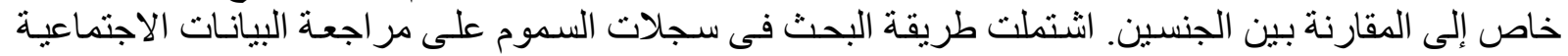

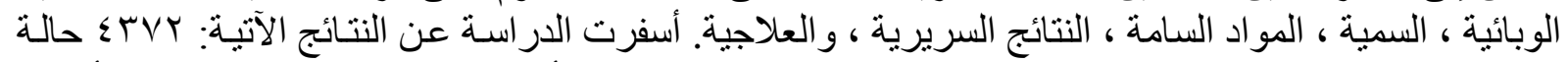

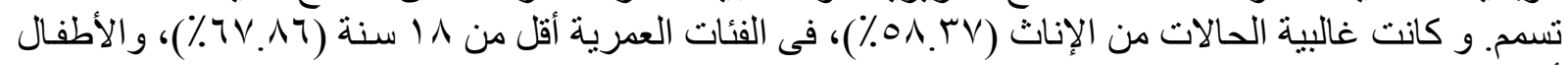

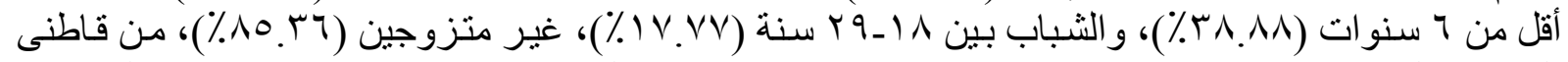

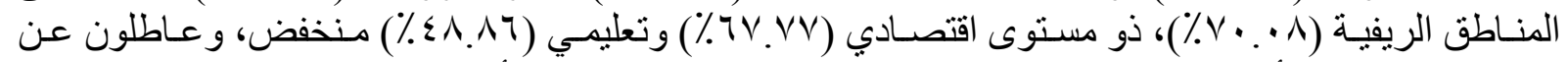

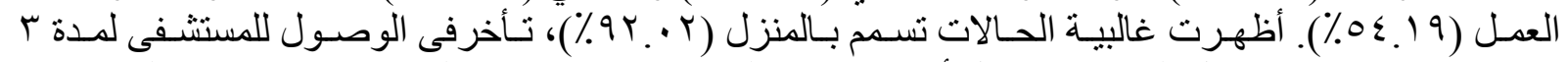

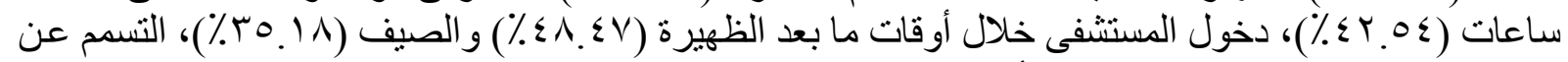

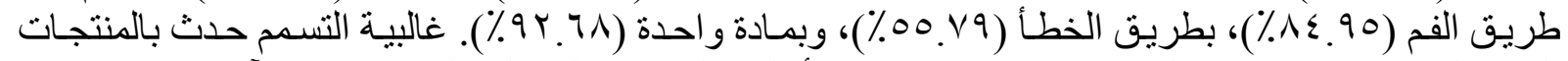

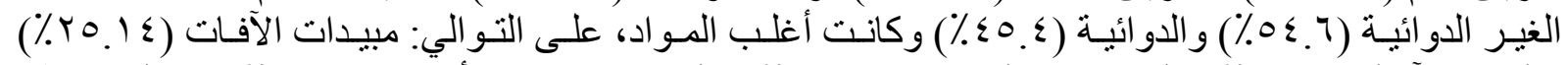

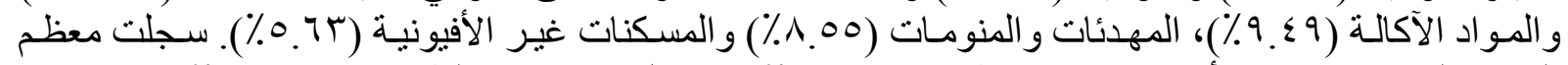

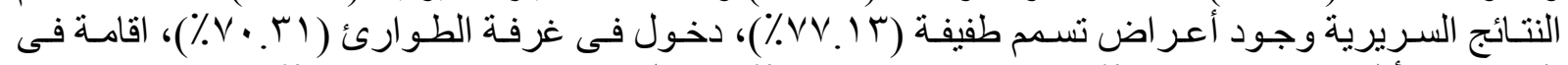

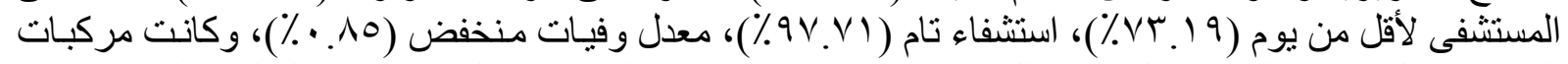

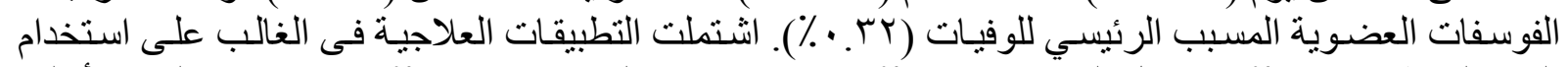

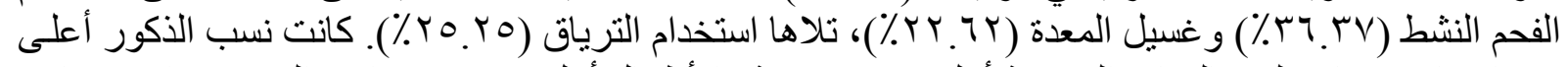

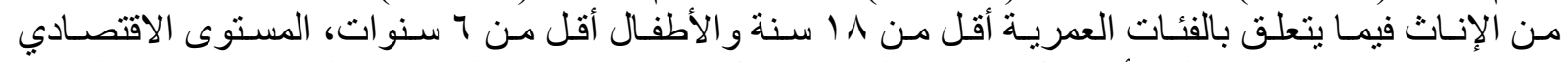

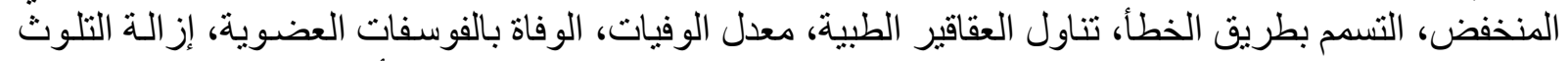

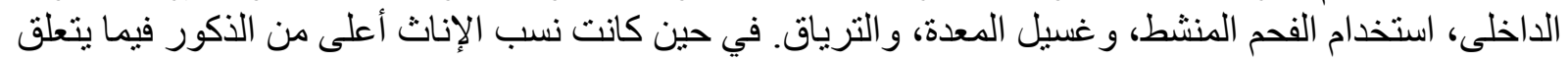

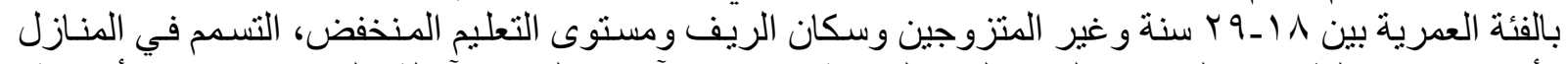

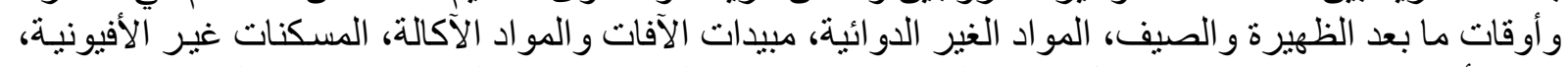

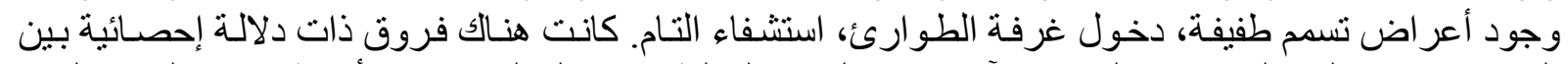

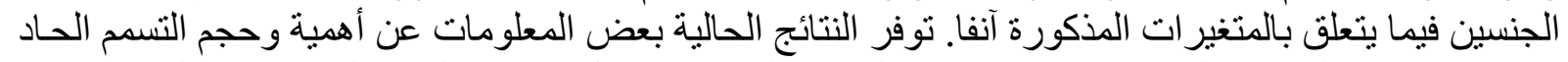

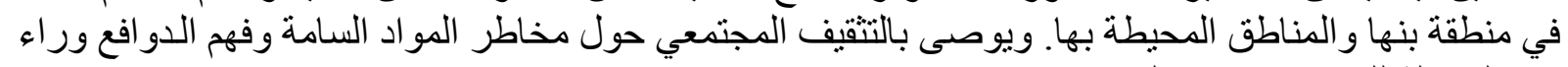
هذّ المشكلة للحد من حدوث التسمح. 